\title{
Universiteit
}

Leiden

The Netherlands

\section{Single organic molecules for photonic quantum technologies}

Toninelli, C.; Gerhardt, I.; Clark, A.S.; Reserbat-Plantey, A.; Goetzinger, S.; Ristanovic, Z.;

... ; Orrit, M.A.J.G.

\section{Citation}

Toninelli, C., Gerhardt, I., Clark, A. S., Reserbat-Plantey, A., Goetzinger, S., Ristanovic, Z., ... Orrit, M. A. J. G. (2021). Single organic molecules for photonic quantum technologies. Nature Materials, 20, 1615-1628. doi:10.1038/s41563-021-00987-4

Version:

Publisher's Version

License:

Licensed under Article 25fa Copyright Act/Law (Amendment Taverne)

Downloaded from: https://hdl.handle.net/1887/3254614

Note: To cite this publication please use the final published version (if applicable). 


\title{
Single organic molecules for photonic quantum technologies
}

\author{
C. Toninelli $\oplus^{1,2} \llbracket$, I. Gerhardt ${ }^{3}$, A. S. Clark ${ }^{4}$, A. Reserbat-Plantey ${ }^{5}$, S. Götzinger ${ }^{6,7}$, Z. Ristanović ${ }^{8}$, \\ M. Colautti ${ }^{12}$, P. Lombardi ${ }^{1,2}$, K. D. Major ${ }^{4}{ }^{4}$, I. Deperasińska9 ${ }^{9}$, W. H. Pernice ${ }^{10}$, \\ F. H. L. Koppens $\circledast^{5,11}$, B. Kozankiewicz $\oplus^{9}$, A. Gourdon $\circledast^{12}{ }^{12}$, V. Sandoghdar $\circledast^{6,7}$ and M. Orrit ${ }^{8}$
}

Isolating single molecules in the solid state has allowed fundamental experiments in basic and applied sciences. When cooled down to liquid helium temperature, certain molecules show transition lines that are tens of megahertz wide, limited by only the excited-state lifetime. The extreme flexibility in the synthesis of organic materials provides, at low costs, a wide palette of emission wavelengths and supporting matrices for such single chromophores. In the past few decades, their controlled coupling to photonic structures has led to an optimized interaction efficiency with light. Molecules can hence be operated as single-photon sources and as nonlinear elements with competitive performance in terms of coherence, scalability and compatibility with diverse integrated platforms. Moreover, they can be used as transducers for the optical read-out of fields and material properties, with the promise of single-quanta resolution in the sensing of charges and motion. We show that quantum emitters based on single molecules hold promise to play a key role in the development of quantum science and technologies.

M odern societies have an ever-growing need for efficient computation techniques and for fast and secure communication, to distribute a huge amount of data around the globe. By harnessing quantum effects present at the nanoscale, new quantum technologies can be employed to meet these needs, including quantum cryptography and fully fledged quantum information processing. In addition, the perturbation that the environment has on a quantum system can be exploited to create new sensing devices that provide unprecedented sensitivity and resolution and can in principle be deployed within large networks.

The generation and manipulation of quantum states of light is required for key applications, such as photonic quantum simulation $^{1,2}$, linear optical quantum computing ${ }^{3}$, device-independent or long-distance quantum key distribution protocols ${ }^{4}$, subshot-noise imaging ${ }^{5}$ and quantum metrology $y^{6}$. In this context, single impurities in solid-state systems can act as bright, on-demand single-photon sources, which are a crucial resource for photonic quantum technologies. Quantum emitters may also perform as nonlinear elements at the few-photon level ${ }^{7}$ and as nanoscale sensors, allowing the optical read-out of local properties of materials and fields. Among others, single molecules in the solid state offer competitive and reliable properties, with several key advantages. First, they are very small and have well-defined transition dipole moments, so they can be used as nanoscopic sensors for a number of scalar and vector quantities such as pressure, strain, temperature, electric and magnetic fields, as well as optical fields. Second, organic molecules can be designed and synthesized for different parts of the visible spectrum and integrated in hybrid devices, a feature that can be a limiting factor for sources naturally embedded in semiconductor materials.
Third, the combination of small size and ease of fabrication makes organic molecules ideal for applications where high densities and scalability are desirable. Fourth, organic dye molecules can have strong zero-phonon lines, which reach their Fourier-limited natural linewidth at liquid helium temperature, thus providing very bright and stable sources of photons with among the highest degree of coherence in the solid state.

Although many of these features have been known since the early 90s, owing to the development of specific, efficient light-molecule interfaces and hybrid molecular devices in the past ten years, molecular quantum emitters are making the jump from proof of principle to the era of complex quantum optics experiments and multiphoton devices.

Here we review the recent advances of single-molecule studies for quantum technologies, with a special focus on the coupling to nanophotonic structures for the enhancement and control of their interaction with quantum light. We discuss how this can extend the quantum optical toolbox for molecules, enabling flexible and efficient quantum photonic devices and new diverse applications.

In the 'Basics of single-molecule photophysics' section, we discuss some fundamentals of molecules and their photophysics (see also refs. ${ }^{8-10}$ ). Some key experiments of the past decade are then summarized, with single molecules acting as well-isolated single quantum systems: we review the advances in the generation of non-classical states of light in the 'Single-molecule-based single-photon sources' section, while nonlinear light-matter interactions at the single-molecule level are reported in the 'Single-molecule optical nonlinearities' section. The 'Molecule-photon interfaces' section highlights recent results in the integration of molecules into

\footnotetext{
${ }^{1}$ CNR-INO, Sesto Fiorentino, Italy. ${ }^{2}$ LENS, European Laboratory for Nonlinear Spectroscopy, Sesto Fiorentino, Italy. ${ }^{3}$ Institute for Quantum Science and Technology (IQST) and 3rd Institute of Physics, Stuttgart, Germany. ${ }^{4}$ Centre for Cold Matter, Blackett Laboratory, Imperial College London, London, UK. ${ }^{5}$ ICFO - Institut de Ciencies Fotoniques, The Barcelona Institute of Science and Technology, Castelldefels, Spain. ${ }^{6}$ Max Planck Institute for the Science of Light, Erlangen, Germany. ${ }^{7}$ Friedrich-Alexander University of Erlangen-Nürnberg, Erlangen, Germany. ${ }^{8}$ Huygens-Kamerlingh Onnes Laboratory, LION, Leiden, The Netherlands. ${ }^{9}$ nstitute of Physics, Polish Academy of Sciences, Warsaw, Poland. ${ }^{10}$ Physikalisches Institut, Westfälische Wilhelms, Universität Münster, Münster, Germany. "ICREA - Institució Catalana de Recerca i Estudis Avançats, Barcelona, Spain. ${ }^{12}$ CEMES-CNRS, Toulouse, France. 凶e-mail: toninelli@lens.unifi.it
} 


\section{Box 1 | General principle of single-molecule photophysics}

Many organic molecules have electronic transitions in the spectral range from 200 to $800 \mathrm{~nm}$. This transition is associated with the light-induced dipole moment (panel a), and has a strength of a few Debye. Fluorescence is the emission of light due to electronic transitions between singlet states following an absorption event, with typical excited-state lifetimes of a few nanoseconds. A vibrational manifold accompanies the electronic levels (panel b) and part of the emission is redshifted compared with the excitation light, meaning that fluorescence can be separated with standard optical filters. Differences in the vibrational potential of the electronic ground and excited states mean that the motion of the nuclei is indeed coupled to the electronic transitions. The larger the similarity between the nuclear positions in the ground and excited state, the stronger the transition probability for the so-called 0-0 ZPL that does not involve molecular vibrations. In the linear approximation, the Franck-Condon factors account for the relative intensity of the different vibro-electronic transitions, associated with a given harmonic vibrational mode. Room-temperature single-molecule spectroscopy with ultrafast laser pulses can unveil the coherence between these levels ${ }^{108,109}$. Inter-system crossing (ISC) (panel b) is a process that competes with fluorescence. Allowed by spin-orbit coupling, it is a weak interaction in planar molecules as long as no heavy atoms are involved. The molecule is then trapped in a long-lived triplet state until it decays by emission of phosphorescence, or by internal conversion. ISC is important not only in solar cells and display technology but also for the electrical excitation of single molecules ${ }^{110}$. Single molecules can be embedded as substitutional impurities in crystalline matrices with diverse fabrication processes, such as sublimation or reprecipitation $^{107}$ (c.f. panel c). Maximizing the fluorescence quantum yield and emission brightness often requires that the excited states of the host matrix, singlet and triplet, are located energetically above the guest's excited singlet state ${ }^{111}$. In these solid materials, the electron-phonon coupling with the host system gives rise to a broad phonon wing in the emission spectrum, redshifted with respect to the common mode of the $0-0 \mathrm{ZPL}^{102}$. The Debye-Waller factor is defined as the ratio of the intensity emitted into the $0-0$ ZPL to the total emitted intensity and decays exponentially with temperature ${ }^{9}$, resulting in a negligible emission in the $0-0 \mathrm{ZPL}$ for molecules at ambient conditions. Such coupling with phonons also induces the so-called Stokes shift between the energy of the maximum in the absorption and emission spectrum. Moreover, second-order phonon interactions bring about dephasing of the induced dipole, causing a temperature-dependent broadening of the $0-0$ transition ${ }^{102,103}$. For certain guest-host systems and very rigid molecules under cryogenic conditions (below $2 \mathrm{~K}$ ), the $\mathrm{ZPL}$ narrows down to its natural linewidth (few tens of megahertz, c.f. panel d). Although the individual guest molecules are nominally all identical, their transition frequencies are influenced by local charges and strain in their environment, resulting in a residual inhomogenous broadening that ranges from a few gigahertz in high-quality sublimation-grown crystals (panel e) ${ }^{112}$ to thousands of gigahertz in non-equilibrium crystallizations ${ }^{58}$ or amorphous hosts such as polymers ${ }^{79}$. a

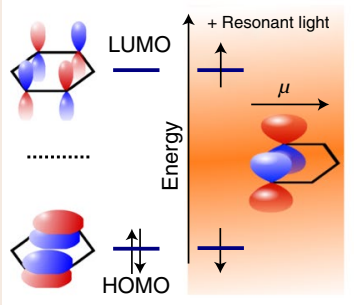

b

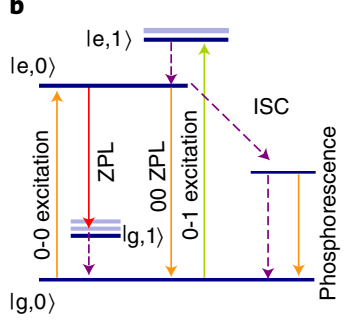

c

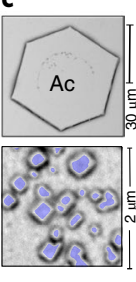

d

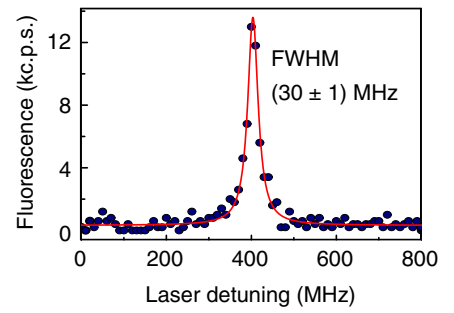

e

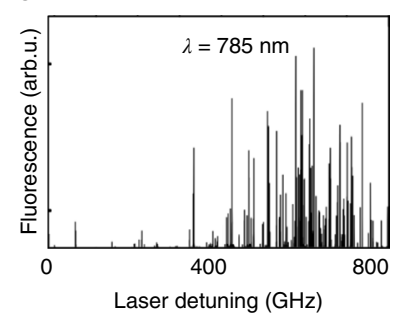

Single-molecule photophysics. a, Artistic view of aromatic molecular orbitals in a single time frame (colours correspond to the phase sign), before and after (white and orange-shaded area) the coupling by a resonant light field, inducing an oscillating dipole (moment $\mu$ ). LUMO and $\mathrm{HOMO}$ stand for the lowest unoccupied and the highest occupied molecular orbital, respectively. b. Simplified Jabłoński diagram showing the molecular energy levels relevant in the fluorescence process. The eigenstate notation describes the excitation of both the electronic and the vibrational degrees of freedom (just one mechanical mode is depicted for clarity). Dashed lines correspond to non-radiative transitions. c, Scanning electron microscope images of Ac crystals obtained via sublimation and reprecipitation ${ }^{46}$. d, Fluorescence excitation spectrum of a single DBT molecule in naphthalene at $1.4 \mathrm{~K}$ revealing the Lorentzian profile of the ZPL, with a Fourier-limited linewidth, full-width at half-maximum FWHM=30 MHz. e, Fluorescence excitation spectrum of an Ac crystal doped with DBT, showing the expected inhomogenous broadening (few hundred gigahertz) around the central wavelength $\lambda$. Credit: panel c adapted with permission from ref. ${ }^{46}$, American Chemical Society.

photonic structures for efficient light extraction and the development of integrated architectures for experiments with increased complexity. In the 'Single-molecule sensing and quantum sensing' section, we present progress in single-molecule sensing towards quantum sensing, and finally discuss future applications and an outlook for molecular quantum technologies.

\section{Basics of single-molecule photophysics}

Since the first demonstration of single-molecule fluorescence detection at cryogenic temperature ${ }^{11}$, this almost background-free technique has enabled single-molecule experiments even at room temperature and in a wide spread of disciplines, such as biology ${ }^{12}$ and materials science ${ }^{13}$. In the domain of quantum optics, where a high degree of coherence and thus lifetime-limited emission is crucial, planar and rigid polycyclic aromatic hydrocarbons (PAHs) are of particular interest because their linewidths tend to be dramatically reduced at temperatures below $2 \mathrm{~K}$ ( ref. $^{9}$; Box 1$)$. This sets them apart from molecules such as rhodamines and coumarins, which are very common fluorescent labels for room-temperature experiments, but display broad lines under cryogenic conditions. The most commonly used guest $\mathrm{PAH}$ molecules include pentacene, terrylene (Tr), perylene, dibenzanthanthrene (DBATT) and dibenzoterrylene (DBT), whose chemical structures are shown in Fig. 1a. Host molecules are often chemically saturated (they 


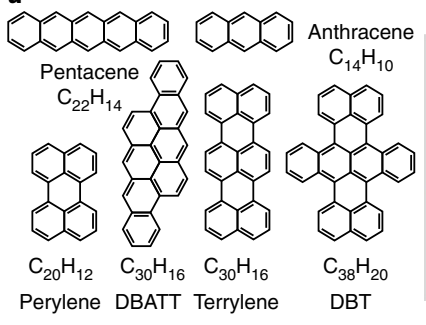

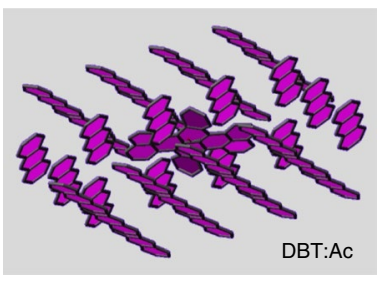

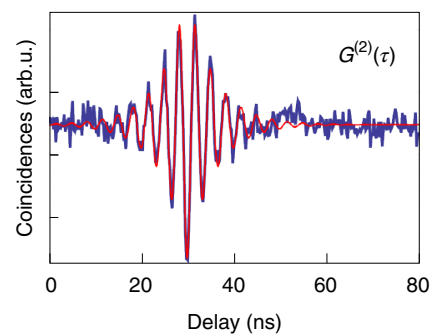

c

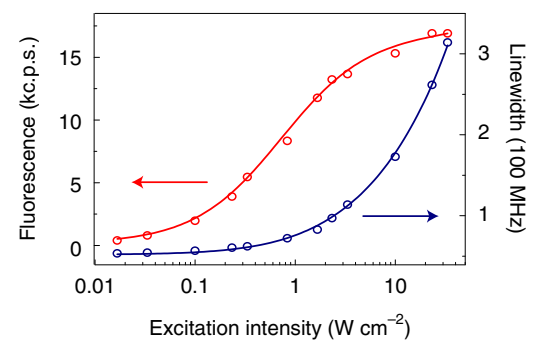

Fig. 1 | Polyaromatic hydrocarbons. a, Chemical structures of several PAH molecules used in quantum technologies. Right: DFT calculation for the Ac crystal structure with a DBT molecule replacing three host molecules (Gaussian 09 package). $\mathbf{b}$, The coherence of the electronic ZPL transition allows for the observation of several Rabi cycles, shown in the un-normalized autocorrelation function, $G^{(2)}(\tau)$, obtained from a start-stop measurement. arb.u., arbitrary units. c, Power dependences of the fluorescence emission and of the ZPL linewidth from a DBT molecule ${ }^{107}$. Credit: panel $\mathbf{c}$ adapted with permission from ref. ${ }^{107}$, American Chemical Society.

present only single C-C bonds), but can also be light PAHs such as substituted benzene, terphenyl, naphthalene, anthracene (Ac) and some of their derivatives (see also Box 2). The molecular insertion of the guest in the host lattice, corresponding to the minimum free energy, can also be calculated by molecular mechanics. An example is sketched in Fig. 1a for DBT in Ac. Once inserted into a suitable host, these fluorescent guest molecules exhibit a small Stokes shift and a fluorescence quantum yield close to unity ${ }^{14}$. Notably, for rigid dye molecules such as Tr, DBATT and DBT, the emission into the 0-0 zero-phonon line (ZPL) at low temperature occurs with a probability exceeding $50 \%$. Moreover, photochemistry is usually absent, so they are expected to be indefinitely photostable under normal illumination conditions ${ }^{15}$.

Under resonant excitation (0-0 excitation, shown as an orange arrow in panel b of the figure in Box 1) of the electronic transition at cryogenic temperature, redshifted photons are usually detected after spectral rejection of the pump light. The decay of the excited state into the vibrational manifold of the electronic ground state also implies that consecutive emitted photons are not coherent. However, the coherence of the molecules' internal states results in Rabi oscillations ${ }^{16}$, which are visible for instance in the autocorrelation function shown Fig. 1b. When the frequency of a narrow-linewidth laser is tuned across the transition, the detected fluorescence counts display a typical Lorentzian line shape, associated with the exponential spontaneous decay of the excited state. As the illumination intensity of the laser is increased, the excitation line broadens and the peak count rate saturates (Fig. 1c). The single-photon nature of the emitted light was further explored in ref. ${ }^{17}$, where resonance fluorescence was observed together with the well-known Mollow triplet. Pulsed excitation of a molecule has also allowed coherent state preparation, and up to $11 \pi$ Rabi cycles were observed $^{18}$.

When lifetime-limited photons are required, it is possible to use a cross-polarization configuration to suppress the excitation light ${ }^{17}$, or excite the molecule into a higher vibrational level of the electronic excited state. This so-called 0-1 excitation allows for the generation of spectrally narrow-band photons, which have the linewidth of the 0-0 transition ${ }^{19-22}$. This is particularly relevant for the generation of coherent, indistinguishable photons, discussed in the next section.

\section{Single-molecule-based single-photon sources}

In this section, we focus our discussion on the use of molecules as single-photon sources based on the figures of merit introduced in Box 3. In the condensed phase, single molecules were the first systems to show single-photon statistics. This was observed for pentacene in $p$-terphenyl at liquid helium temperatures as early as $1992^{16}$. Later, these observations were extended to different molecules in liquids and on surfaces at ambient conditions $\mathrm{s}^{23-25}$. The state-of-the-art performance of molecule-based single-photon sources is summarized in the table in Box 3, which shows values competitive with the best available solid-state sources. More details for other systems can be found in recent papers that review single-photon sources based on quantum $\operatorname{dots}^{26}$, point defects in wide bandgap materials ${ }^{27}$ and two-dimensional semiconductors ${ }^{28}$.

Several common PAH molecules display a near-unity quantum yield (see ref. ${ }^{14}$ ), can achieve very low values for the second-order autocorrelation function at zero time delay $\left(g^{(2)}(0)=0.00(3)\right.$ $\left(\right.$ ref. $\left.\left.{ }^{29}\right)\right)$ and are hence competitive with other solid-state systems (Fig. 2a). They also show negligible intersystem crossing (ISC), meaning that they exhibit minimal blinking. In the case of DBT in Ac, for instance, the ISC probability at low temperature is as low as $10^{-7}$ (ref. ${ }^{30}$ ). This source, emitting in the near-infrared at around $785 \mathrm{~nm}$, is stable both at room ${ }^{31,32}$ and at cryogenic ${ }^{33}$ temperatures with an off-time below a per cent. A time trace with microsecond binning time, recorded at ambient conditions from a single Tr molecule in a $p$-terphenyl crystal is shown as an example in Fig. 2 b. Such a source, combined with smart photonic engineering, yields an extremely regular stream of single photons $s^{34}$, leading to intensity squeezing (ratio of intensity fluctuations with respect to the shot noise) of up to $2.2 \mathrm{~dB}$ at room temperature.

The collection efficiency is typically the most limiting factor in the overall loss budget for molecule-based single-photon sources (see table in Box 3). Simple photonic structures (see 'Moleculephoton interfaces' section) can improve collection efficiencies from a few per cent with low numerical optics, up to $20 \%$ into a single spatial mode even in cryogenic experiments ${ }^{35}$.

Near-unity collection efficiency in ambient conditions has been demonstrated for the combination of a planar antenna with high-numerical-aperture optics ${ }^{36}$, yielding just under 50 million photons per second at room temperature. Count rates exceeding a few million counts per second have been demonstrated at cryogenic temperatures ${ }^{35}$ (Fig. 2c), reaching the order of ten million counts per second for a single molecule coupled to a plasmonic nanoantenna ${ }^{37}$. Considering a ZPL branching ratio of up to $50 \%$ from the product of the Debye-Waller and Franck-Condon factors, starting from an excited-state lifetime of about $5 \mathrm{~ns}$, a $100 \%$ collection efficiency would correspond to a count rate of 100 million counts per second at the first lens. Such values for the collection efficiency are within reach in certain photonic structures, as discussed in the 'Molecule-photon interfaces' section. Molecule-based single-photon sources can also be operated under pulsed excitation $^{25,32,33}$, and recent efforts to optimize collection efficiency by coupling molecules to photonic structures can be readily extended to the pulsed domain. 


\section{Box 2 | The choice of a chromophore}

The number of molecules so far studied for single-molecule photophysics and quantum applications in particular is somehow small but certainly could increase in the next decade. Most experiments have been limited to a few PAHs: pentacene, perylene, Tr, DBT, DBATT, as shown in Fig. 1a, and perylene bisimide. The constraints on the design of new molecular emitters appropriate for quantum technologies are rather strong, as follows.

(1) Suitable electronic properties, as discussed in the 'Basics of single-molecule photophysics' section, in particular strong transition probability into the ZPL and small Stokes shift, which implies rigid molecules. Indeed, apart from one exception ${ }^{39}$, all single molecules with strong Fourier-limited ZPLs known so far are extremely rigid and flat PAHs.

(2) Absorption wavelengths in the red to infrared to fit usual laser wavelengths and to reduce fluorescence from impurities and optics. In the case of PAHs, these small HOMO-LUMO gaps are obtained by increasing the number of fused benzene rings while limiting the number of Clar sextets ${ }^{113}$. Further extension of the delocalized $\pi$-system, such as in oligorylenes ${ }^{114,115}$ or oligoacenes, can lead to absorbances in the infrared around $1 \mu \mathrm{m}$. This wavelength range is interesting for coupling with silicon photonics and for quantum communications in general. However, molecules absorbing at such wavelengths present low fluorescence efficiencies as a consequence of the energy gap law ${ }^{116}$. For smaller HOMO-LUMO gaps, fewer vibrational quanta are involved in internal conversion, and the Franck-Condon factors become exponentially larger. Creation of a few $\mathrm{C}-\mathrm{H}$ (or N-H, O-H) stretch quanta competes efficiently with spontaneous emission. A possible remedy is to deuterate the molecules, to lower the vibrational quantum of $\mathrm{C}-\mathrm{D}$ ( or N-D, O-D) bonds. Another solution to this difficulty could be to substitute peripheral hydrogen atoms by other rigid substituents such as halides ${ }^{117}$.
Another challenging point is that reducing the HOMO-LUMO gaps increases the reactivity of molecules towards photo-oxidation or dimerization. For instance, acenes longer than pentacene only exist as dimers in solution.

(3) Negligible spectral diffusion implying that these PAHs cannot be substituted by non-rigid groups with internal degrees of freedom (see remark in (1) above). This constraint is a severe one, as the addition of bulky and flexible substituents is the way to improve the solubility of rigid planar molecules by increasing the intermolecular distances, hence reducing the $\pi-\pi$ stacking, and also to protect them from further reactions. This lack of solubility of unsubstituted PAHs implies that their synthesis, and often that of their precursors, is carried out in harsh conditions, that the purification steps are problematic, often limited to washing and sublimation when possible, and that standard spectroscopic characterizations such as NMR in solution are limited.

A possible alternative new way of studying large, insoluble, electronically delocalized and reactive unsubstituted PAHs is first to prepare soluble and chemically stable non-planar precursors that can be synthesized, fully purified and characterized by standard in-solution organic chemistry techniques. These compounds can then be diluted in host materials. In the final step, the volatile protecting groups can be removed by ultraviolet irradiation, yielding the final PAHs as shown in the figure in Box 2 for the case of long acenes ${ }^{118-120}$. In such an example, the precursors are non-planar carbonylated compounds, comprising small units of large HOMO-LUMO gaps such as naphthalene or Ac subunits. The presence of the bridging carbonyl group prevents strong intermolecular $\pi-\pi$ interaction and breaks the electronic coupling between subunits. After dilution in a matrix, photodeprotection removes volatile carbon monoxide leaving isolated insoluble PAHs.

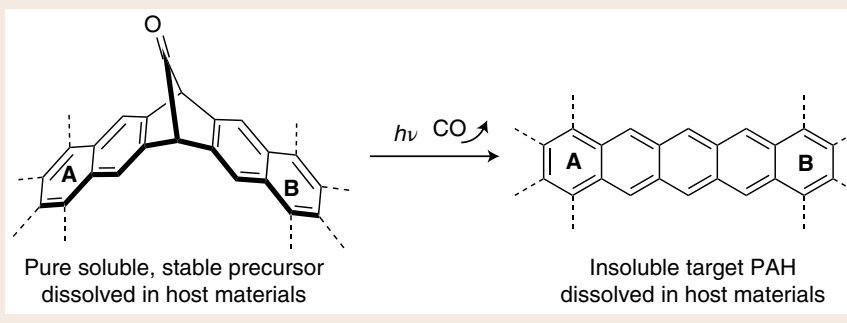

In-situ preparation of a PAH diluted in a solid matrix by photochemical decarbonylation of a precursor.

Under cryogenic conditions, both 0-0 excitation and 0-1 pumping schemes can provide lifetime-limited emission ${ }^{22,29}$. The ultranarrow linewidth of the 0-0 ZPLs in PAHs, typically only a few tens of megahertz wide (Box 1), offer a unique advantage for the efficient coupling of single photons to other emitters or even to cold atomic systems. In ref. ${ }^{38}$, for example, interaction of photons originating from a single DBATT molecule with sodium atoms in the gas phase is demonstrated (Fig. 2d). Moreover, in the case of very rigid matrices and well-behaved PAH emitters, negligibly small spectral diffusion can be achieved even with simple sample preparation procedures. For example, a dilution of DBT in melted, polycrystalline naphthalene or in host matrices with halogen heteroatoms leads to highly stable ZPLs with nearly no spectral diffusion. Figure 2e shows real-time excitation spectra of DBT in 2,3-dibromonaphthalene, illustrating high spectral stability over about $20 \mathrm{~min}$. The sensitivity of the ZPL frequency to temperature and pressure, leading to possible fluctuations, are extensively discussed in the 'Singlemolecule sensing and quantum sensing' section.

The first experiments on two-photon interference (TPI) ${ }^{20,39}$ were later enhanced by using an atomic filter on the emission of a single DBATT molecule. A crucial parameter is the visibility, given by the normalized difference of the $g^{(2)}(0)$ in the case of interfering versus non-interfering photons (Box 3$)^{29}$. There, a visibility of $0.94 \pm 0.03$ was achieved (Fig. 2f). Recently TPI from a single DBT molecule has also been demonstrated under pulsed excitation ${ }^{40}$. TPI has also been observed for photons from two remote molecules ${ }^{19}$ (Fig. 2g). This impressive result, which pioneered such measurements in solid-state quantum emitters, was made possible by the local tunability of molecules through the Stark effect ${ }^{41}$. Finally, two independent photons were entangled using a beam splitter, proven by a Bell violation in the raw data of $2.24 \pm 0.12$ (ref. ${ }^{42}$ ). It is worth noticing that, unlike most cases in literature, the attained purity of the 


\section{Box 3 | Figures of merit of quantum-emitter-based single-photon sources}

The property of emitting one photon at a time, ideally on demand, is relevant for many quantum technology applications. Any background alters the photon statistics and spoils the performance of such single-photon-based devices. The single-photon purity is the ratio of the probability of generating a single photon, $p_{1}$, over the joint probability of there being any photon event $\sum_{n} p_{n}$, where $p_{n}$ is the probability of detecting $n$ photons ${ }^{121}$. This is commonly estimated by measuring the autocorrelation of the emitted light, $g^{(2)}(\tau)$, yielding an upper bound to purity as $1-g^{(2)}(0) / 2$. Moreover, for low mean photon number, the following relationship holds $g^{(2)}(0)=2 p_{2} / p_{1}^{2}$. For an ideal single-photon emitter, $g^{(2)}(0)=0$, a feature referred to as 'anti-bunching, and the purity is 1 .

A further important and related figure of merit of a single-photon source is its efficiency, often termed brightness. The efficiency with which a single quantum emitter can generate photons in a known optical mode depends on a number of factors, including the efficiency of excitation (absorption cross-section), the quantum yield of fluorescence and the overall collection efficiency. Each of these terms has to be optimized. By employing 0-1 excitation, for instance, the population of the molecule can be fully inverted at low temperature, corresponding to unity excitation efficiency ${ }^{122}$. The product of the efficiency and the rate at which one can repeatedly excite the system gives a number of photon counts per second. For single molecules, this is comparable to the best single-photon sources, as detailed in the main text.

A crucial property for certain application is the ability of the emitted photons to undergo TPI, when impinging on separate input ports of a 50:50 beam splitter. This so-called Hong-OuMandel interference effect has been used to enable precision relative timing measurements ${ }^{123}$ and is the central technique to enable Bell-state analysis for quantum teleportation ${ }^{124,125}$, as well as LOQC schemes ${ }^{3}$.
The visibility for two coalescing photons in Hong-Ou-Mandel interference is defined as $V=\left(g_{\perp}^{(2)}(0)-g_{\|}^{(2)}(0)\right) / g_{\perp}^{(2)}(0)$, with $g_{\|, \perp}^{(2)}(0)$ being the autocorrelation function in zero for parallel and orthogonal photons. However, this definition holds only for zero-time delay and the time window where quantum interference takes place might be very small, as the dip width is given by the coherence time of the emitter. In the case of Fourier-limited emission, the entire photon wavepacket can interfere, and all emitted photons are usable. In pulsed operation, visibility is estimated calculating directly the integral of the autocorrelation function over the pulse duration, obtaining as an upper bound $\bar{V}=\tau_{\mathrm{c}} / 2 \tau_{\mathrm{r}}$, with $\tau_{\mathrm{r}}$ being the radiative decay time and $\tau_{\mathrm{c}}$ the coherence time, affected by the excess dephasing rate $\Gamma^{\star}$, as $1 / \tau_{\mathrm{c}}=1 /$ $\left(2 \tau_{\mathrm{r}}\right)+\Gamma^{\star}$. Here then, perfect indistinguishability corresponds to the so-called lifetime-limited linewidth condition, which typically requires cryogenic operation. It should be noticed that, for some solid-state systems, the emission of photons with high wavepacket overlap is hindered also by frequency fluctuations on timescales larger than the inverse linewidth and up to seconds, often called spectral diffusion. Furthermore, the remote interference of two quantum emitters can be used to prepare them in a joint entangled state. Following the discussion above, the possible applications of molecules as single-photon sources depend critically on the operating conditions: room temperature versus low temperature. Quantum technologies that can benefit from room-temperature molecular sources are those that rely on the peculiar statistics of single-photon Fock states (subshot-noise imaging or quantum random number generators), on the superposition principle and on the no-cloning theorem (certain QKD protocols). When two-photon interference (LOQC, Boson sampling, more complex QKD protocols) or narrow-band photons are required (coupling with atomic memories), then low-temperature operation becomes necessary.

Figures of merit characterizing molecule-based single-photon sources

\begin{tabular}{|c|c|c|c|c|}
\hline \multirow{2}{*}{$\begin{array}{l}\text { Figure of merit } \\
\text { Collection efficiency and } \\
\text { maximum count rate }\end{array}$} & \multirow{2}{*}{$\begin{array}{l}\text { Definition and measurement strategies } \\
\text { Probability that a generated photon is } \\
\text { collected per trigger event and maximum } \\
\text { count rate at the detector }\end{array}$} & \multicolumn{2}{|c|}{ State of the art for molecules } & \multirow{2}{*}{$\begin{array}{l}\text { References } \\
34,36\end{array}$} \\
\hline & & RT & Free space (NA 1.65): >96\%, & \\
\hline & & RT & Waveguide: 20\% & 47 \\
\hline & & LT & $\begin{array}{l}\text { Free space (NA 0.67): 40\%, 4 } \\
\text { Mphotons per second }\end{array}$ & 35 \\
\hline & & LT & Waveguide: 5\% & 60 \\
\hline \multirow[t]{2}{*}{ Single-photon purity } & $\begin{array}{l}p_{1} / \sum_{n} p_{n} \rightarrow 1-g^{(2)}(0) / 2 \\
\text { Second-order correlation function }\end{array}$ & $\mathrm{RT}$ & $g^{(2)}(0)=0.03_{-0.01}^{+0.01}$ & 107 \\
\hline & & LT & $g^{(2)}(0)=0.00_{-0.00}^{+0.03}$ & 29,35 \\
\hline \multirow[t]{2}{*}{ Linewidth } & Resonant laser scans or tunable filtering & RT & $\sim 30 \mathrm{THz}$ & 31 \\
\hline & & LT & $\sim 10-100 \mathrm{MHz}$ & 126 \\
\hline \multirow[t]{2}{*}{ Indistinguishability } & Two-photon interference visibility & $1 \mathrm{~mol}$ & $\sim 95 \%, \sim 80 \%$ (95\% at $1.4 \mathrm{~K})$ & 29,40 \\
\hline & & $2 \mathrm{~mol}$ & $\sim 75 \%$ & 19 \\
\hline \multirow[t]{2}{*}{ Tunability } & Voltage-dependent excitation spectra & LT & $\begin{array}{l}300 \mathrm{MHz}\left(\mathrm{kV} \mathrm{cm}^{-1}\right)^{-1} \text { linear, } 0.15 \mathrm{MHz}(\mathrm{kV} \\
\left.\mathrm{cm}^{-1}\right)^{-2} \text { quadratic, } 400 \mathrm{GHz} \text { tuning range }\end{array}$ & 45 \\
\hline & & LT & $1.5 \mathrm{GHz}\left(\mathrm{kV} \mathrm{cm}^{-1}\right)^{-1}$ & 44 \\
\hline
\end{tabular}

Figures of merit include efficiency, single-photon purity, linewidth of the associated radiative transition, photon indistinguishability and frequency tunability. Here we summarize these concepts and the state-of-the-art values for molecules. The count rate at the detector is normalized by the detector quantum efficiency only; RT and LT stand for room temperature and low temperature, respectively, and NA stands for numerical aperture. The maximum count rate detected is not mentioned for the waveguide-integrated molecules because it depends on the out-coupler efficiency and is not relevant here. 

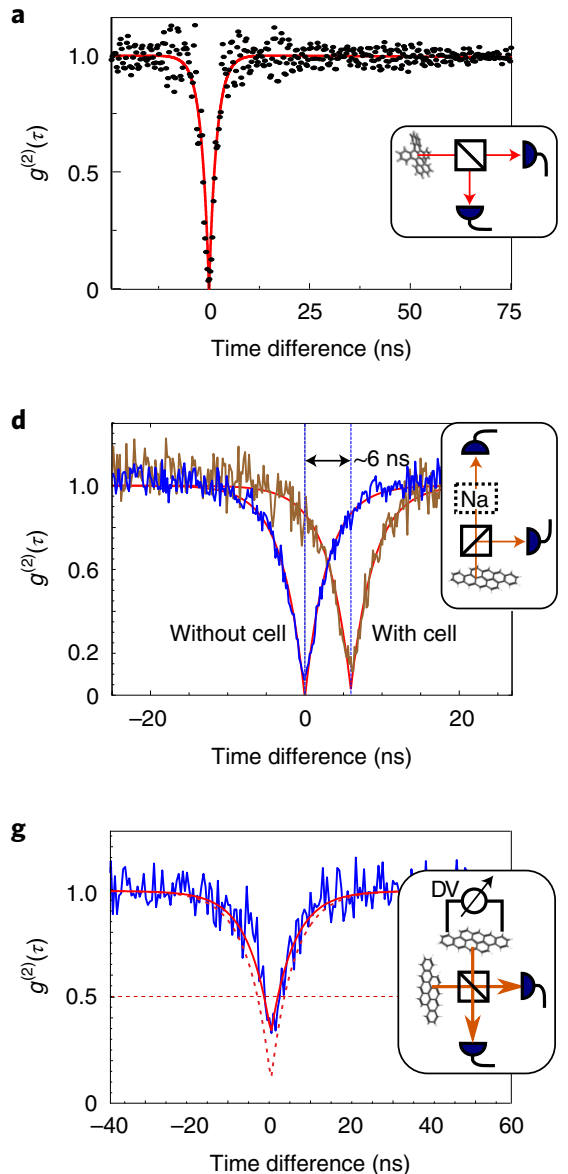
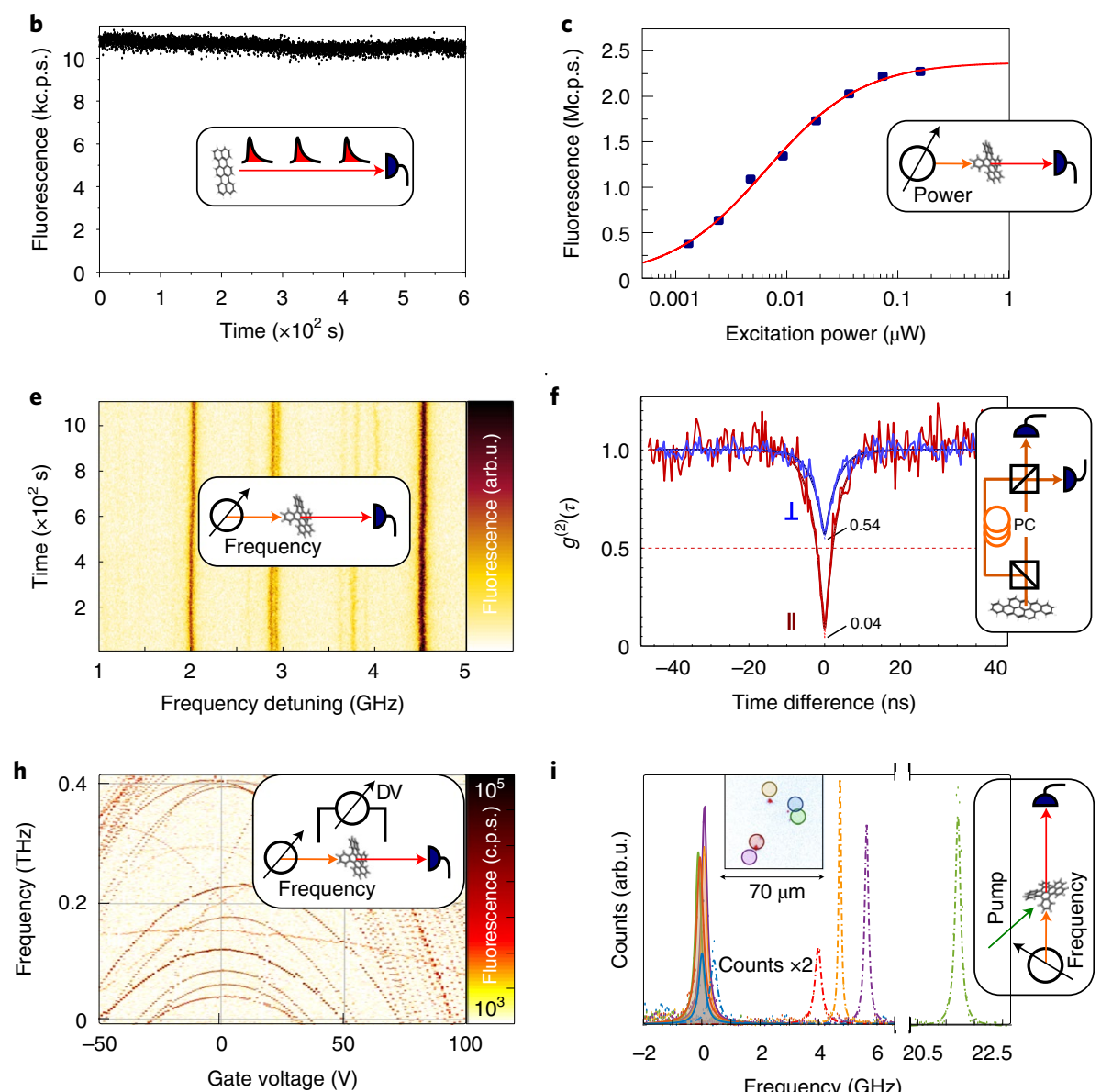

i

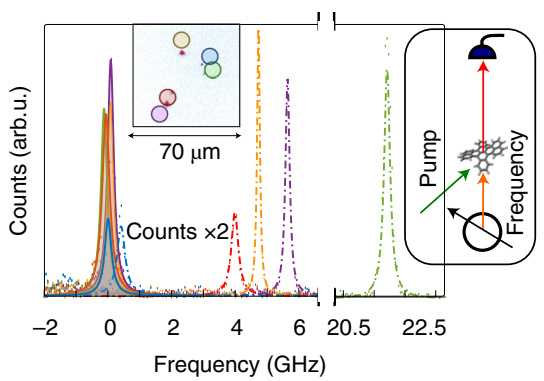

Fig. 2 | Single-molecule-based single-photon sources. a, Example of a second-order autocorrelation function, measured in a Hanbury Brown and Twiss configuration for a single DBT molecule in Ac, upon continuous-wave 0-1 pumping ${ }^{35}$. $\mathbf{b}$, Typical time trace obtained for single-molecule emission at room temperature for $\operatorname{Tr}$ in $p$-terphenyl. c, Saturation curve showing the detected, background corrected counts from a single molecule at low temperature, as reported in ref. ${ }^{35}$. d, Evidence in the second-order autocorrelation function $g^{(2)}(\tau)$ of the interaction between single photons from molecules and atomic systems (sodium, $\mathrm{Na})^{38}$. e, Excitation spectra for DBT molecules in 2,3-dibromonaphthalene ${ }^{44}$ shown in colour scale as a function of time, illustrating negligible spectral diffusion. f, Two-photon interference obtained in a Hong-Ou-Mandel configuration for the photon stream from a single DBATT molecule as reported in ref. ${ }^{29}$. PC, polarization control unit. $\mathbf{g}$, Two-photon interference in the photon streams obtained collecting the ZPLs of two remote single molecules under continuous-wave 0-1 pumping and using and applied Stark voltage (DV) ${ }^{19}$. h. Excitation spectra are reported in colour scale as in e, showing the characteristic second-order Stark shift of DBT molecules, induced by the applied gate voltage (DV). The sample is described in ref. ${ }^{45}$. i, Excitation spectra of five different DBT molecules whose relative position is given in the confocal fluorescence map in the inset ${ }^{46}$. The ZPL frequency of four of them is brought in resonance (within two linewidths) with the fifth one (solid-blue filled line), by means of a superimposed pump beam. Dashed and solid lines refer to the spectra before and after tuning, respectively. The insets to all panels represent the measurement concept and the employed molecular source. Orange and red solid lines correspond to light at the ZPL frequency and in the redshifted channels, respectively ${ }^{46}$. Credit: panels adapted with permission from: a,c , ref. ${ }^{35}$ under a Creative Commons licence CC BY 4.0; d, ref. ${ }^{38}$, Springer Nature Ltd; f, ref. ${ }^{29}$, under a Creative Commons licence CC BY $4.0 ; \mathbf{g}$, ref. ${ }^{19}$, APS; $\mathbf{h}$, ref. ${ }^{45}$, American Chemical Society; $\mathbf{i}$, ref. ${ }^{46}$, American Chemical Society.

photon stream and the indistinguishability of molecular photons do not require optical cavities.

Depending on the internal structure, the Stark effect is generally found to be linear for molecules without inversion symmetry, or quadratic for inversion-symmetric molecules ${ }^{43}$. However, local imperfections in the host matrix can lift the degeneracy, such that a linear Stark shift is observed even in nominally centrosymmetric impurities. In this way, a sensitivity of $1.5 \mathrm{GHz}\left(\mathrm{kV} \mathrm{cm}^{-1}\right)^{-1}$ was recently achieved $^{44}$. Naturally, electric charges and fields can be probed by this effect-even on nanoscopic length scales, as discussed below. In Fig. 2h, we report in colour scale the excitation spectrum of several DBT molecules in Ac as a function of the applied gate voltage, reaching a total shift of more than $200 \mathrm{GHz}$ (ref. ${ }^{45}$ ). Here DBT:Ac nanocrystals were integrated in a polymer matrix, which formed a substrate for the transfer of a two-dimensional-material electrode.
A new method is emerging to optically tune single-molecule ZPLs without the introduction of any nanofabrication step ${ }^{46}$. This is based on an additional pump beam that locally induces a persistent electric field as a function of the applied dose. This approach has allowed the resonances of up to five emitters to be matched within twice their linewidth (Fig. 2i), all within an area of about $50 \mu \mathrm{m}$. With a resolution mostly determined by the laser spot size, it appears particularly suitable for molecular emitters integrated on chips ${ }^{47}$.

The molecular systems presented here are state-of-the art single-photon sources and excel in terms of optical coherence time, intensity and frequency stability of the photon stream and also with respect to the possibility of interfering photons from distinct molecules. These features, combined with the ease of fabrication, tuning and integration will be relevant for interfacing flying qubits 
a

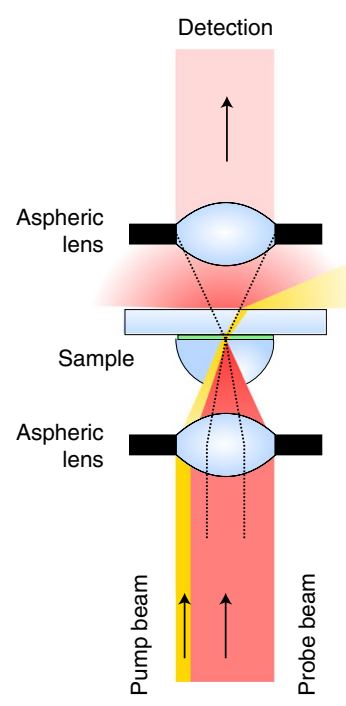

b

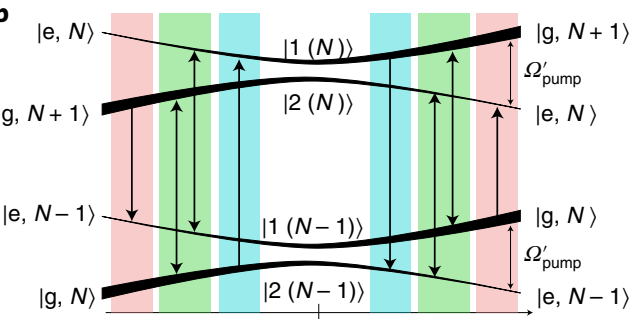

0

Pump frequency detuning $\Delta$

d

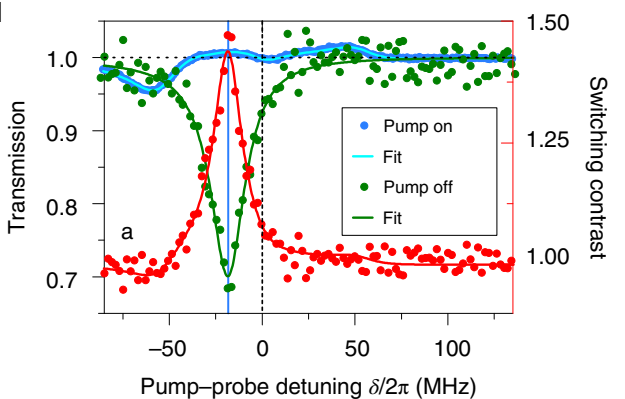

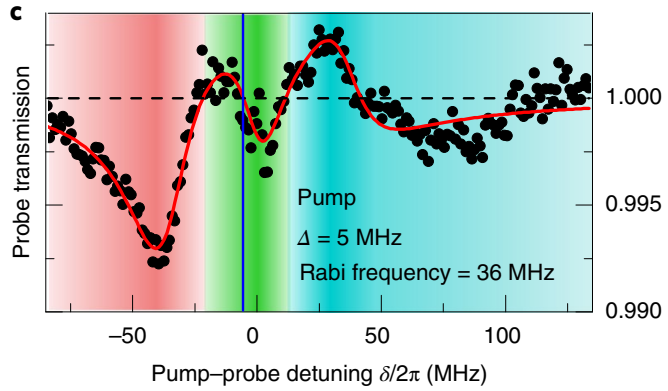

e

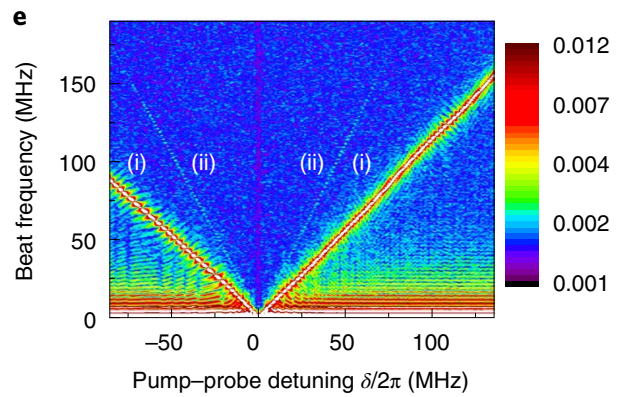

Fig. 3 | A single molecule acting as a nonlinear medium. a, Sketch of a setup for transmission measurements on a single molecule subjected to superimposed probe and pump beam, inducing a nonlinear behaviour. $\mathbf{b}$, Simplified level scheme of a single molecule under the influence of a strong pump beam. Shown are the new eigenstates of the combined molecular resonance and the pump beam, as a function of the pump detuning $(\Delta)$. The thickness of the energy levels indicates the difference in population. The colours indicate the transitions at probe frequency $\omega_{\text {pump }}-\Omega^{\prime}{ }_{\text {pump }}$ (red), $\omega_{\text {pump }}$ (green) and $\omega_{\text {pump }}+\Omega_{\text {pump }}^{\prime}$ (blue). $\Omega_{\text {pump }}^{\prime}$ and $\omega_{\text {pump }}$ are the pump generalized Rabi and angular frequency, respectively. c, Transmitted power of a weak probe beam. The underlying colours indicate different nonlinear processes. d, Switching the transmission of the probe beam with a weak pump beam. The red curve is obtained as the ratio between the probe transmission with pump on and off. e, Fourier transform of the time-dependent measurement recorded in transmission. The colour shading indicates the magnitude of the Fourier transform of the transmission signal on a logarithmic scale. The straight line marked with (ii) is the result of a four-wave mixing process. The generated light interferes with the probe beam creating a beat frequency, besides the expected beating of pump and probe marked with (i). Credit: panels adapted with permission from: b, ref. ${ }^{51}$, APS; c-e, ref. ${ }^{52}$, Springer Nature Ltd.

with atomic quantum memories, as well as for speeding-up multiphoton devices, hence enabling the implementation of small-scale linear optical quantum computers (LOQC) and long-distance quantum key distribution (QKD).

\section{Single-molecule optical nonlinearities}

In recent years, the desire to control photons with photons has flourished, spurred on by the ever-increasing demand for low-power classical transistors and novel potential applications of optical quantum information processing. Photon-photon interactions can be effectively mediated by matter. If the interaction between light and matter is strong enough, a single photon can saturate a single emitter such that the reaction of the system to one or two photons is very different. The large extinction cross-section of a single molecule at cryogenic temperatures, together with the possibility to tightly focus a laser beam using high-refractive-index solid immersion lenses, offers the possibility to observe saturation at the few-photon level. About a decade ago, it was demonstrated that a single molecule is sufficient to reflect about $20 \%$ of a weak probe laser beam and can act as an optical transistor ${ }^{48}$. A setup for transmission measurements is depicted in Fig. 3a. The situation changes when a second pump laser excites the molecule into a vibrationally excited state of the first electronic excited state. The intensity of the transmitted probe light is now dependent on the power of the pump laser. For larger excitation powers, stimulated emission even amplifies the transmitted laser beam. Many pump photons are required in this configuration to control the transmitted laser beam, due to the low absorption cross-section of the vibrationally excited state. Ultimately, the goal is to control one photon using a second photon, via the controlled interaction with a single molecule. The key to this goal is the strong nonlinearity described above, combined with nanophotonic structures, such as those discussed in the 'Moleculephoton interfaces' section.

The use of two beams with different frequencies gives access to other nonlinear effects beyond simple saturation. Effects like the a.c. Stark shift, stimulated Rayleigh scattering and the hyper-Raman effect were first observed with ensembles of atoms ${ }^{49}$ and later by monitoring the fluorescence, even with a single molecule ${ }^{50}$. All these effects can be understood in the dressed-atom picture, where the pump laser induces a split of the levels by the effective Rabi frequency. The populations of the new dressed eigenstates depend on the detuning of the pump laser with respect to the transition of the two-level system ${ }^{51,52}$.

Figure $3 \mathrm{~b}$ shows the splitting of the two levels into doublets that are separated by the generalized Rabi frequency, which is a function of the pump-beam intensity. If such a system is now probed by a weak beam in a transmission type of experiment, there are three possibilities to connect the four levels (marked in blue, green and red). Note that the thickness of the new levels indicates the population of these new eigenstates. Figure $3 c$ shows a transmission spectrum where a pump beam with a generalized Rabi frequency of $36 \mathrm{MHz}$ is detuned by $5 \mathrm{MHz}$ with respect to the bare molecule transition $^{51}$. All the expected features are clearly visible and marked for clarity with the respective colours.

For a probe frequency equal to $\omega_{\text {pump }}-\Omega_{\text {pump }}^{\prime}$, where $\Omega^{\prime}$ pump and $\omega_{\text {pump }}$ are the pump generalized Rabi and angular frequency, respectively (red-shaded areas), the population in the lower state exceeds the population in the upper state and the probe beam experiences extinction in a transmission experiment. However, due to the a.c. Stark effect, this will occur at a lower frequency compared with 
a
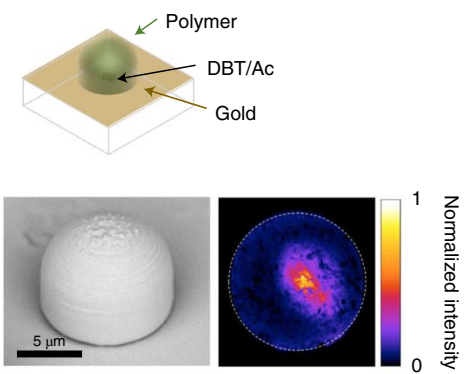

d
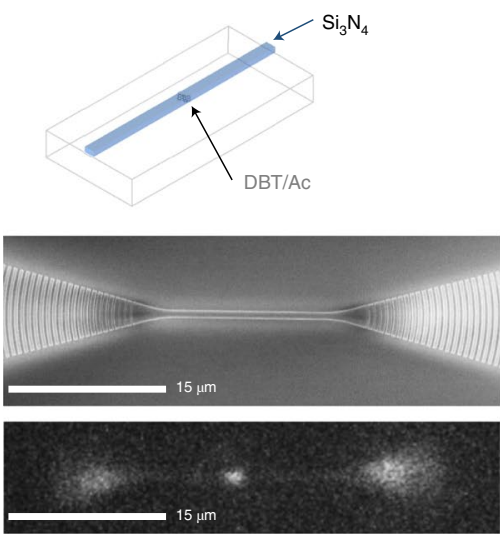

g

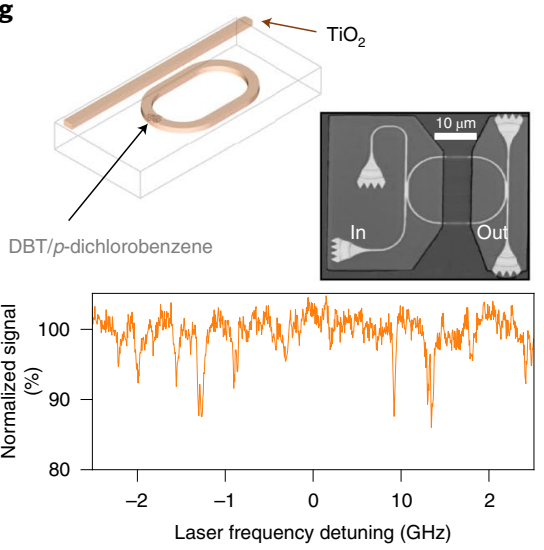

b
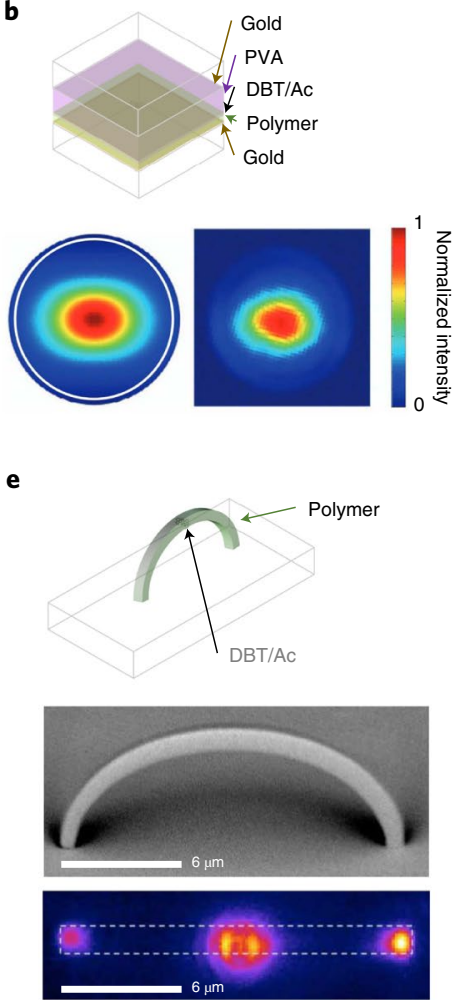

h

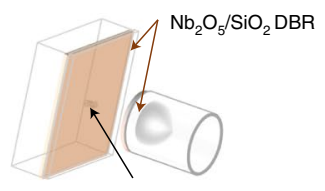

$\mathrm{DBT} / \mathrm{Ac}$

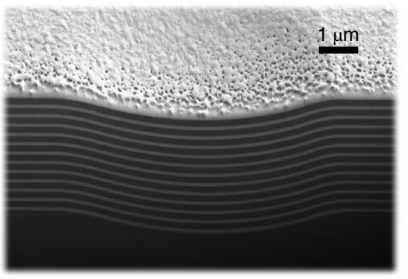

c
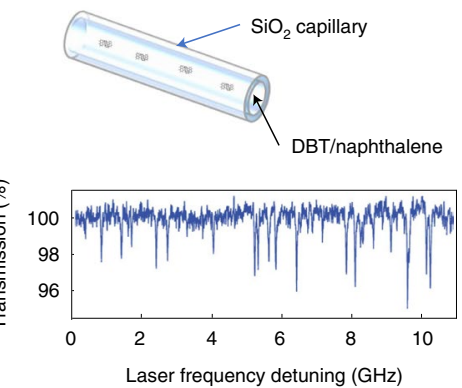

f
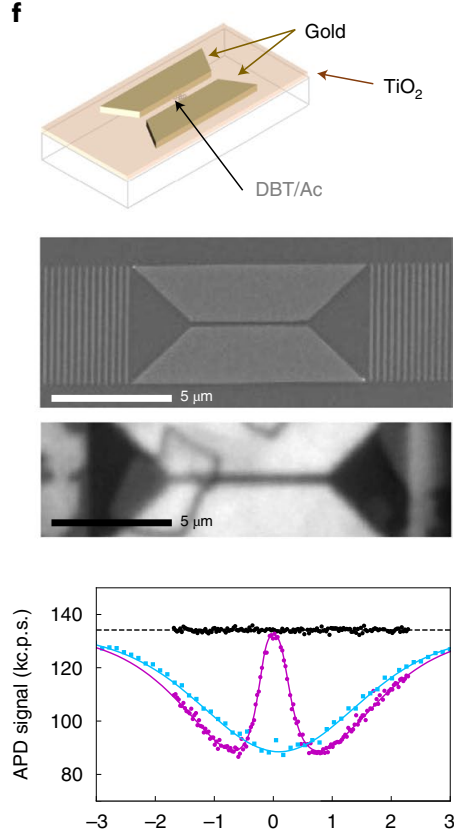

Laser frequency detuning $(\mathrm{GHz})$

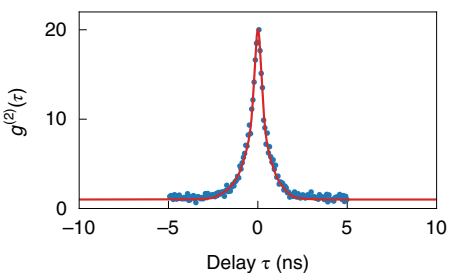

Fig. 4 | Molecule-photon interfaces. a, Top: nanofabricated solid immersion lens on gold, used to extract photons from a DBT molecule in an Ac nanocrystal. Bottom left: scanning electron micrograph (SEM) of the structure. Bottom right: back-focal plane image of DBT fluorescence ${ }^{35}$. b, Top: a planar dielectric antenna with layers of gold, polymer, DBT-doped Ac, PVA (polyvinyl alcohol) and a thin gold director layer. Bottom left: simulated angular emission spectrum. Bottom right: back-focal plane image of DBT fluorescence ${ }^{55}$. c, Top: silica $\left(\mathrm{SiO}_{2}\right.$ ) nanocapillary filled with DBT-doped naphthalene. Bottom: transmission through the capillary as the laser frequency is tuned, showing pronounced extinction dips ${ }^{58}$. $\mathbf{d}$, Top: a silicon nitride $\left(\mathrm{Si}_{3} \mathrm{~N}_{4}\right)$ nanowire waveguide for coupling single molecules to the evanescent field of the guided mode. Middle: an SEM image of the fabricated device with gratings. Bottom: fluorescence from a DBT molecule coupled to the waveguide, imaged with an electron multiplying charge coupled device (EMCCD) camera ${ }^{47}$. e, Top: a suspended polymer waveguide containing a DBT-doped Ac nanocrystal. Middle: SEM image of the structure. Bottom: fluorescence from an ensemble of DBT molecules, imaged from the underside on an EMCCD camera ${ }^{35}$. $\mathbf{f}$, Top: hybrid plasmonic waveguide consisting of gold islands with a varying gap on the surface of $\mathrm{TiO}_{2}$ layer ${ }^{68}$. Middle: SEM image of a fabricated device with grating couplers. Bottom: white light microscope image of DBT-doped Ac crystals on the device. $\mathbf{g}$, Top: nanophotonic $\mathrm{TiO}_{2}$ ring resonator used to couple to DBT molecules in para-dichlorobenzene, which cover parts of the ring. Middle: SEM image of the fabricated device with input/output waveguides. Bottom: resonant extinction dips as in c, but with higher visibility due to cavity-enhanced coupling ${ }^{69}$. h, Top left: open-access microcavity formed between flat and rounded distributed Bragg reflectors (DBRs) ${ }^{15}$. Bottom left: SEM image of the DBR on the rounded mirror. Top right: laser reflection from the cavity when it is detuned from both molecule and cavity (black), when it is tuned across the cavity with the molecule detuned (blue), and when both cavity and molecule are resonant with the laser (purple). Bottom right: second-order correlation function of the emitted light from the cavity, showing strong photon bunching. APD, avalanche photodiode. Credit: panels adapted with permission from: a,e, ref. ${ }^{35}$ under a Creative Commons licence CC BY 4.0; b, ref. ${ }^{55}$, Springer Nature Ltd; $\mathbf{c}$, ref. ${ }^{58}$, APS; $\mathbf{d}$, ref. ${ }^{47}$, American Chemical Society; f, ref. ${ }^{68}$, AIP; $\mathbf{g}$, ref. ${ }^{69}$, IOP; $\mathbf{h}$, ref. ${ }^{15}$, Springer Nature Ltd. 
a

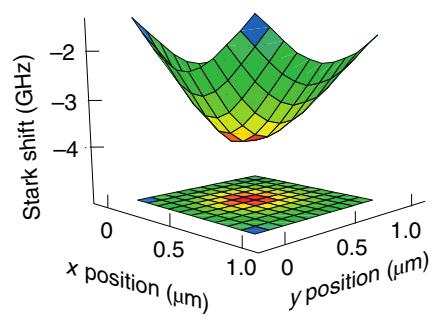

d

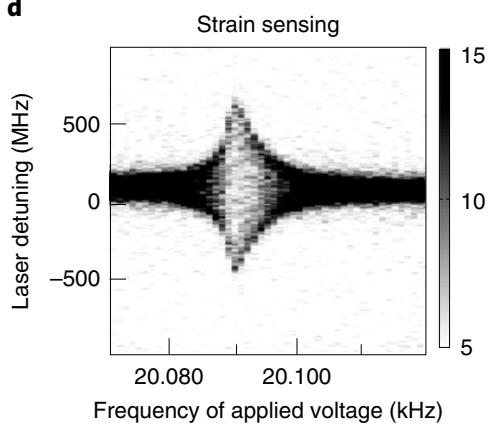

b

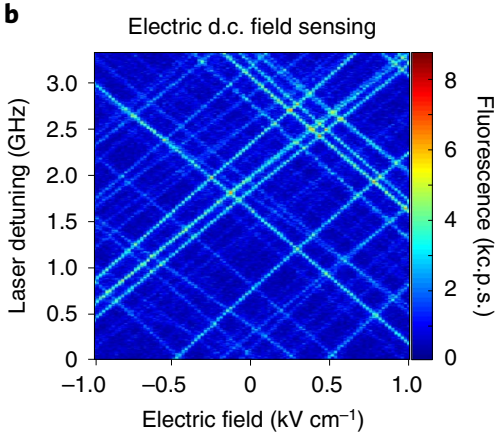

e

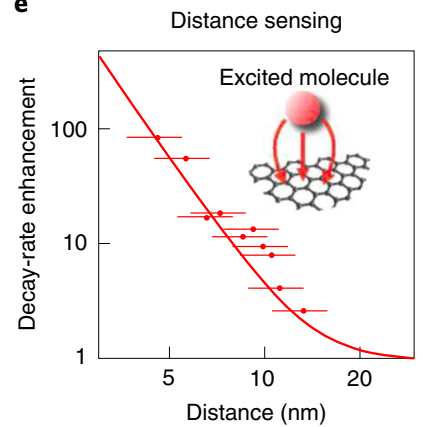

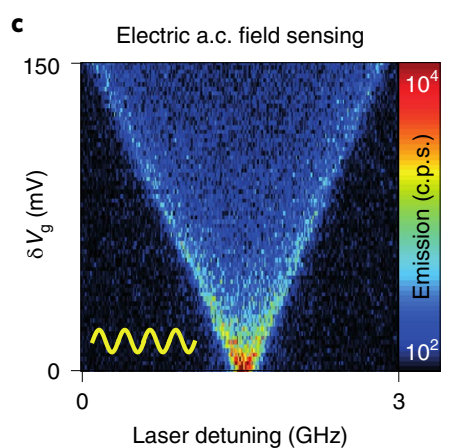

f

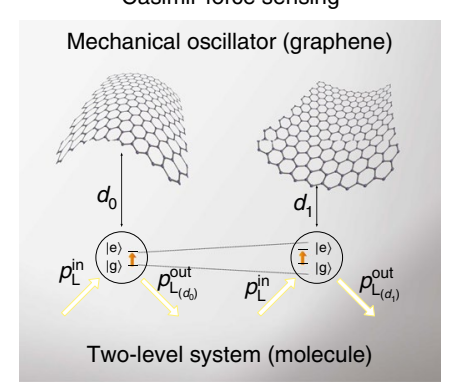

Fig. 5 | Using single molecules for quantum sensing. a, Scanning probe microscopy to identify separation and coupling between two Tr molecules spaced by $12 \mathrm{~nm}$. The scanning tip is terminated with a metallic sphere to apply a non-homogeneous electric field ${ }^{77}$. $\mathbf{b}$, Linear Stark shift of DBT single molecules in 2,3-dibromonaphthalene; the external electric field was applied nearly parallel to the $a$ axis of the crystal ${ }^{44}$. c, Dynamic control of a single DBT molecule's energy through an out-of-plane a.c. electric field applied through a graphene electrode $\left(\delta V_{g}\right.$ is the applied a.c. voltage amplitude) ${ }^{45}$. d, Single DBT molecule used as a transducer of local strain field in an Ac crystal attached to a tuning fork, showing the fork's resonance. At the mechanical resonant frequency, the strain is enhanced and the optical line is broadened ${ }^{88}$.e, Non-resonant energy transfer between graphene and rhodamine molecules showing decay rate enhancement at small separation, and consequent quenching of the molecular emission under graphene ${ }^{95}$. $\mathbf{f}$, Theoretical proposal for sensing the mechanical motion of a graphene resonator via vacuum potential. The molecule energy levels are shifted depending on the distance to the membrane. $|e\rangle$ and $|g\rangle$ are the excited and the ground states of a two-level system, and $p_{\mathrm{L}}^{\text {in }}$ and $p_{\mathrm{L}}^{\text {out }}$ are the incoming and scattered laser power, respectively. The latter is a function of the distance to graphene $\left(d_{i}\right)^{98}$. Credit: panels adapted with permission from: $\mathbf{a}$, ref. ${ }^{77}, \mathrm{AAAS} ; \mathbf{b}$, ref. ${ }^{44}$, Wiley-VCH; $\mathbf{c}$, ref. ${ }^{45}$, American $\mathrm{Chemical}$ Society; d, ref. ${ }^{88}$, APS; e, ref. ${ }^{95}$, American Chemical Society; f, ref. ${ }^{98}$, APS.

the bare molecular transition. For a probe frequency equal to $\omega_{\text {pump }}$ (green), the population of the connected states is equal and 'stimulated Rayleigh scattering' occurs, resulting in an energy transfer between pump and probe ${ }^{49}$. If the probe beam is tuned further to a frequency $\omega_{\text {pump }}+\Omega^{\prime}$ pump (blue-shaded areas) it will connect two dressed states, where the population of the upper state exceeds that of the lower state. In the asymptotic limit of large detuning, this corresponds to a transition between the dressed states $|\mathrm{g}, \mathrm{N}+1\rangle$ and $|\mathrm{e}, N-1\rangle$, which means that the pump field loses two photons while the probe field gains a photon due to stimulated emission, hence the name three-photon amplification (or hyper-Raman). In this notation, $N$ is the number of photons in the pump field, and $\mathrm{e}$ and $g$ are the excited and ground states of the molecule, respectively.

The a.c. Stark effect can be used to switch the probe beam with about ten photons per lifetime, displaying a nonlinear effect close to the single-photon level. In Fig. 3d, the switching contrast is shown (red line), calculated as the ratio between the probe transmission with pump on and off. A careful analysis of light collected in the transmission channel reveals another interesting nonlinear phenomenon. If the Fourier transform of the recorded signal is calculated for each pump and probe detuning (as shown in Fig. 3e) one observes, besides the beating of pump and probe (i), another signal which originates from the beating of the probe with light generated with a detuning of $2 \delta$ (ii). It stems from a degenerate four-wave mixing process and has about $3 \%$ of the signal strength of the probe beam. These experiments impressively demonstrate nonlinear optical processes due to the coupling of extremely weak laser beams to a single molecule.

The long-sought-after goal of single photon-photon interactions mediated by a molecule is therefore tantalizingly close. The introduction of molecules into nanostructures, discussed in detail in the 'Molecule-photon interfaces' section, can be used to enhance the above described processes and bring us into the regime of creating new quantum devices such as non-demolition photon detectors, deterministic quantum information processing gates and single-molecule-mediated optical switches.

\section{Molecule-photon interfaces}

Although appreciable light-matter interaction can in principle be achieved with strongly focused laser beams in free space ${ }^{17,53}$, tailoring the local electromagnetic field modes available to an emitter is a compact and successful strategy to realize efficient and coherent light-matter interfaces. The relevant parameter describing how much emission from a two-level system is collected into a given mode $(\beta)$ also determines the coherent extinction of photons in that mode by the two-level system. The relative drop in transmission in the absence of dephasing is simply given by $\left(2 \beta-\beta^{2}\right)$. Photonic interfaces are hence crucial for the realization of efficient single-photon sources, as well as enhancing nonlinear interactions at the few-photon level.

Strategies to efficiently extract light from single molecules in free space have evolved from traditional solid-immersion-lens schemes 
with collection efficiencies from $10 \%$ to $20 \%^{54}$, to nanofabricated lenses on reflective surfaces (Fig. $4 \mathrm{a}$ ) addressing a large number of emitters on a single sample ${ }^{35}$ and multilayer dielectric and metallic media, tailored for high extraction efficiency. Because molecular crystals have typically lower refractive index with respect to diamond or inorganic semiconductors, even simple, broadband structures allow for efficient extraction of light. Indeed, under ambient conditions, a collection efficiency of $96 \%$ into a 1.65 -numerical-aperture objective was demonstrated in ref. ${ }^{36}$. Strong directionality from all emitters on the surface was achieved in a planar antenna (Fig. 4b $)^{55}$. Spatial-mode matching between an incoming laser field and the emission from a single molecule was achieved via coupling to tapered optical fibres ${ }^{56}$, fibre facet ${ }^{57}$ and subwavelength dielectric capillaries $^{58}$ (Fig. 4c), with efficiencies around $10 \%$ in all cases.

On-chip integration of single molecules represents another important prospect, enabling quantum light sources, processing units and single-photon detectors ${ }^{59}$ to be fabricated on the same integrated platform. Individual components have already been demonstrated. These include single molecules evanescently coupled to $\mathrm{Si}_{3} \mathrm{~N}_{4}$ and titanium dioxide $\left(\mathrm{TiO}_{2}\right)$ waveguides ${ }^{47,60,61}$, according to the theoretical proposal from ref. ${ }^{62}$. In Fig. 4 d, a fluorescence map from a single DBT molecule on a ridge waveguide is reported, showing light at the output grating couplers, from which the coupling efficiency is estimated to be around $40 \%{ }^{47}$.

Also, very promising is the direct integration of molecule-doped nanocrystals in polymeric photoresists, which can be structured around selected emitters, either with electron-beam lithography ${ }^{63,64}$ or by direct laser writing techniques ${ }^{35,65}$ (Fig. 4e). Nanoparticles containing single molecules have also been deposited by ink-jet printing $^{66}$. We note in passing that these methods allow for deterministic positioning of molecules and can hence in principle be scaled up to couple several emitters together on the same chip.

Research was carried out also on hybrid plasmonic-dielectric waveguides, which were proposed to improve also single-molecule optical switching ${ }^{67}$ beyond the free-space realization ${ }^{48}$. More recently, single molecules were added to hybrid devices, where a coupling efficiency of around $12 \%$ was achieved to a propagating mode $^{68}$ (Fig. 4f). Such devices can make promising additions to dielectric waveguides, enabling regions of strong light-matter interaction.

Resonant structures can be employed to further enhance the coupling to a single mode. A cavity can also promote decay on the ZPL while suppressing emission through other decay paths, thus enhancing this branching ratio. The coupling of DBT molecules to a nanophotonic $\mathrm{TiO}_{2}$ ring resonator was recently demonstrated in ref. ${ }^{69}$. A coupling efficiency to the cavity mode of $\sim 22 \%$ was found (Fig. 4g), limited by inhomogeneity in the host crystal causing excess scattering of light out of the device.

Open cavities formed of two independently movable mirrors benefit from the selectively alignment and tuning on a molecule of choice $^{70}$. Enhanced interaction of a laser with a single molecule was indeed observed, to the point where the laser could be extinguished in transmission by up to $99 \%{ }^{15,71}$ (Fig. $4 \mathrm{~h}$ ). In ref. ${ }^{15}$, a coupling efficiency to the cavity mode of $\beta_{\text {cav }}=(97.4 \pm 0.3) \%$ was achieved, and a modified branching ratio on the ZPL of $95 \%$ was estimated, starting from typical values $33 \%$.

We observe that the development of efficient light-matter interfaces for molecules is a relatively recent field of research, whereas a lot of effort has been put in the design of microcavities or in nanostructuring diamond and semiconductor materials for decades. Higher coupling efficiencies to waveguides have been reported, for example, in the case of photonic crystal structures ${ }^{72,73}$. However, it appears that structuring semiconductors below a few hundred nanometres (such as in nanowires or in photonic crystals) results in perturbing charge noise and hence spectral diffusion, thus limiting either the choice of photonic architectures or the type of application.
The results here presented all together demonstrate that an efficient light-matter interaction mediated by cavity structures renders molecules ideal single-photon emitters and nonlinear elements for integrated quantum photonics. The next steps to use this system as an on-demand photon source is to engineer the cavity to decay into a known optical mode, ideally matched to an optical fibre or waveguide, without compromising any cavity enhancement. Multiple such molecule-cavity systems must then be built and show identical emission. Based on the scalable production of identical molecules, such a system promises to reach world record multiphoton generation efficiencies that will enable photonic quantum technologies that surpass anything developed so far.

At the ensemble level, the integration of organic emitters in microcavities has attracted enormous interest from different communities studying the effects of strong coupling on photochemical reactions on an ensemble of molecules ${ }^{74}$ and other polaritonic effects. Coupling molecules to the continuum of modes in one-dimensional waveguides or to single-mode cavities appears also an ideal testbed to study many-body coherent effects, such as super- and subradiance, as a function of positional disorder, coupling efficiency, inhomogeneous broadening and dephasing ${ }^{75,76}$.

\section{Single-molecule sensing and quantum sensing}

Single fluorescent molecules are well established in microscopy and sensing, where they can act as transducers between light and a local field or deformation. Optical read-out allows for contactless measurements, which minimizes system perturbations. Another crucial advantage of single molecules for sensing is their nanoscopic size, fixed position inside a solid-state host matrix and exquisitely narrow lines at low temperature, allowing for high-spatial-resolution sensing. In an electrical equivalent of magnetic resonance imaging, strong electric-field gradients were used to resolve two molecules in three dimensions at a resolution of $2 \mathrm{~nm}$ (ref. ${ }^{77}$; Fig. 5a). There, the massive Stark effect of a nanoscopic probe is harnessed alongside the almost background-free detection under fluorescence excitation of the molecules. Correspondingly, gradients in the optical field can also be sensed by measuring Rabi oscillations ${ }^{78}$.

In the early days of cryogenic single-molecule spectroscopy the narrow-band transitions of a molecule were explored to sense external or internal fields that act on the molecule, applying either d.c. Stark fields $\mathrm{s}^{41,79}$ and a.c. fields $\mathrm{s}^{80-82}$. Internal charges in the host matrix can also result in a spectral shift of the molecule's ZPL. These original observations triggered the idea that a single molecule can act as a transducer, enabling the otherwise impossible optical detection of tiny internal charges, defects and ideally single spins.

Towards the goal of single-charge detection, it was shown that the electronegativity of halogen atoms, combined with the herring-bone crystalline structure of a 2,3-dibromonaphthalene host, can induce large electric dipoles on a centrosymmetric molecule such as $\mathrm{DBT}^{44}$. The homogeneity and the linearity of the Stark effect in this system can be appreciated from the graph in Fig. 5b, where the excitation spectrum of several molecules is plotted as a function of the applied external electric field. With an electric dipole moment change of about $1 \mathrm{D}$, this system would allow the optical detection of single electrons at the electron-emitter distance of at least $100 \mathrm{~nm}$ and by a full linewidth frequency shift ${ }^{83,84}$. Similarly, it was proposed in ref. ${ }^{85}$ that a single-charge displacement can be measured by means of single-molecule dynamic triangulation, with positional accuracy better than $6 \mathrm{pm}$. Recent experiments featuring hybrid devices of single DBT molecules coupled to a top graphene electrode have shown broadband (10,000 linewidths) and fast $(100 \mathrm{MHz})$ emission energy tuning owing to the extremely large electric fields obtained in this geometry and the high conductivity of graphene ${ }^{45}$ (Fig. 5c).

External pressure on molecular crystals at low temperatures is another parameter that acts on single chromophores and causes 
an additional frequency shift of the ZPL. In this way, pressure sensors have been demonstrated with a responsivity of the order of $1 \mathrm{GHz}$ atm $^{-1}$ (ref. ${ }^{86}$ ). By exploiting the coupling of local strain to applied a.c. electric fields, single molecules have been used as nanomicrophones, sensing the local vibrations of a solid ${ }^{87}$.

Similarly, strain coupling has been used to detect deformations of a microtuning fork through the corresponding spectral line shift at low temperatures ${ }^{88}$, as shown in Fig. 5d, which demonstrates the interaction with the mechanical oscillator. Coupling light fields to mechanical motion is at the heart of the growing field of optomechanics, which has led to extraordinary sensitivity at various length scales and frequency domains (from gravitational wave interferometers to photonic nano-optomechanical sensor devices) and has been readily extended to the quantum domain. Despite their advantages (high mass resolution, high force sensitivity, large scalability), the transduction of motion in nanomechanical systems remains a challenge. It can be overcome by coupling them with single molecules in the photon-counting regime. A theoretical proposal based on the strong electric-field gradient at the tip of a carbon nanotube moving in the near field of a molecule would enable efficient transduction of the nanomotion as well as a process for optomechanical cooling of the resonator ${ }^{89}$. Recent theoretical advances suggest that the molecule can act as a topological actuator to control the quantum-mechanical spectrum ${ }^{90}$.

Compared with electric fields, the sensitivity of transitions between spinless states of single molecules to magnetic fields is limited, as only a weak second-order diamagnetic Zeeman effect can be monitored $^{91}$. Indeed, no unpaired electrons are available in the usual single molecules. Such experiments are superseded by a large number of experiments on spin-active defect centres, for instance, the nitrogen-vacancy centre in diamond. However, the triplet levels of closed-shell molecules are sensitive to first-order Zeeman interactions, and enable spin manipulations by microwave frequen$\operatorname{cies}^{92,93}$. There, even coherent oscillations can be generated ${ }^{94}$. The combination of the excellent optical properties of single molecules with electronic and nuclear-spin manipulations in their triplet states would open a rich field for quantum information technologies. The challenge here resides in the difficulty to calculate and measure the singlet-triplet energy spacing, which requires broadly tunable laser systems due to the large range of computational predictions. Such laser sources are nowadays available and such investigation is ongoing. The subsequent coherent control of transitions between singlet and triplet states also has yet to be demonstrated, but will no doubt benefit from the interaction of molecules with nanostructures such as those described in the 'Molecule-photon interfaces' section.

Recently, non-resonant energy transfer from single molecules to graphene has been used to define a universal ruler, depending only on fundamental constants, with the emitter lifetime strongly affected by the presence of a single graphene layer ${ }^{95,96}$ (Fig. 5e). Such a universal dependence on distance enables precision localization (up to $5 \mathrm{~nm}$ ) of single DBT molecules in Ac crystals deposited on top of graphene ${ }^{96}$, by measuring the molecule's fluorescence lifetime.

The experiments mentioned so far rely on the specific features of molecules, but most interactions were classical. However, single-molecule sensing can attain single-excitation sensitivity. When a single electron, or a single vibrational quantum is involved, the description becomes quantum mechanical. Entanglement and coherent state transfer between different quantum systems can then be envisaged. In a recent theoretical proposal, for instance, the use of molecules in waveguides is proposed to interface superconducting qubits with optical photons in a hybrid device ${ }^{97}$. Another exciting theoretical proposal that testifies the potential future impact of molecules in quantum sensing is described in ref. ${ }^{98}$, where harnessing vacuum forces (such as Casimir-Polder interaction) is suggested to dispersively couple a single molecule to the motion of a graphene nanomechanical resonator (Fig. 5f). As an example, at a distance of $20 \mathrm{~nm}$ between the graphene resonator and a DBT molecule, the frequency shift of the DBT molecule is 100 times larger than its natural linewidth. Due to this large coupling, the emitter can be used as a transducer to monitor the motion of the graphene in real time, or to squeeze the mechanical resonator position.

\section{Challenges and outlook}

After three decades of hard work on single-molecule studies, their development as efficient and coherent single-photon sources ${ }^{6,29,35,42}$, the successful demonstration of single-photon-single-molecule interactions ${ }^{15}$ including single-photon nonlinearities ${ }^{52}$ and their engineering as sensitive probes ${ }^{44,77,88}$, we are about to embark on an exciting new era. Here, one aims to construct more complex on-chip architectures, where a controlled number of quantum emitters provide different functionalities for a realm of applications in classical and quantum optical technologies. Molecules coupled to photonic nano- and microstructures, such as plasmonic nanoantennas, subwavelength waveguides and microresonators, will be particularly promising for exploiting quantum cooperative effects and many-body phenomena that involve linear or nonlinear interactions ${ }^{75,99}$. Furthermore, quantum optical devices such as nanosensors based on novel quantum-sensing schemes ${ }^{89,98}$, quantum transducers ${ }^{97}$ or multiple sources of non-classical light are within reach.

One of the hurdles to unlock these applications is the control of materials at the single-emitter level. While physical manipulations of single molecules were reported as early as the late 1990s, nanometre control in coupling single molecules to photonic structures remains a topic of current research ${ }^{35,66}$. Future work will have to develop chemical and physical methods to tailor organic and hybrid materials with single-molecule precision. These efforts will be crucial for a number of exciting quantum technological applications, where the efficiency of light-matter interaction phenomena plays an important role ${ }^{100}$

Another central matter of concern, as for any solid-state optical emitter, is host matrix interactions. Indeed, it is the influence of phonons that limit many molecular properties such as the quantum yield, decoherence, absorption cross-section and spectral stability. To tame these unwanted interactions, one is often obliged to perform cryogenic measurements. Despite their key role, however, phonon couplings have often been avoided in solid-state quantum optics rather than scrutinized. Future studies will aim at investigating the 'optomechanical' degrees of freedom both within a single organic molecule (vibrational transitions) and with its environment (optical and acoustic phonons) ${ }^{101-103}$. In addition to minimizing unwanted couplings to improve the degree of coherence at elevated temperatures, there is promise for exploiting the rich phononic landscape of molecules as a resource.

Many of the protocols developed for quantum information processing rely on so-called $\lambda$ configurations, where two long-lived ground states are connected via an excited state. However, common organic dye molecules possess only one ground state because their electron orbitals are composed of paired electrons, so that the ground state is typically a singlet state with no net electronic spin. Here further investigations, of radicals, for example, could open new doors to molecular systems with novel optical properties, including coupling to spins in doublet or triplet ground states. Furthermore, such chemical developments would enable magnetic field sensing at the nanoscale. It is worth noting that besides our focus on PAH molecules as interfaces for photons, there exists a broader molecular quantum ecosystem that comprises species, such as organometallic complexes or stable organic and metal-organic frameworks, with addressable electronic spin states (for a review, see ref. ${ }^{104}$ ). These systems attract attention as potential candidates for long-lived quantum memories coupled to superconducting qubits ${ }^{105}$, as well as for quantum information processing ${ }^{106}$. The advantages of 
molecular platforms are again a bottom-up tailored design and the synthesis scalability.

Organic molecules are often associated with insufficient photostability as their most commonly known applications are in the pigment industry and fluorescence microscopy, where photobleaching is omnipresent. In the context discussed here, this impression is unfounded in many ways because optical excitation of organic molecules in the $1-2 \mathrm{eV}$ energy range is far from the direct photodamage threshold, and photostability is only endangered if chemical reactions are possible. Chemical modifications are strongly inhibited at cryogenic temperatures. Moreover, such long-term photostability can be attained also at room temperature, for samples encapsulated under a controlled atmosphere. Indeed, while the use of organics for large display screens seemed unthinkable at the end of the 1990s, controlled fabrication and packaging have allowed the industry to overcome the photostability issue. Similar engineering can be envisaged for single-molecule-based quantum devices.

Future high-performance optical quantum technologies will most likely require hybrid platforms to benefit from the best features (optical, electrical, magnetic, mechanical and so on) of various materials and qubits. Considering their favourable properties, such as high quantum yield, ease of fabrication and handling, small size and availability at different wavelengths, as well as brightness and high degree of coherence, molecules are ideally suited for such endeavours ${ }^{97}$. What is, indeed, particularly intriguing is that a molecule is intrinsically a hybrid system in which electronic and nuclear degrees of freedom provide a broad range of transition frequencies in the optical, infrared and microwave domain. The exciting path towards harnessing molecules in quantum technologies will most certainly also involve new discoveries that we might be overlooking today.

Received: 9 October 2020; Accepted: 17 March 2021;

Published online: 10 May 2021

\section{References}

1. Aspuru-Guzik, A. \& Walther, P. Photonic quantum simulators. Nat. Phys. 8, 285-291 (2012).

2. Zhong, H.-S. et al. Quantum computational advantage using photons. Science 370, 1460-1463 (2020).

3. Knill, E., Laflamme, R. \& Milburn, G. A scheme for efficient quantum computation with linear optics. Nature 409, 46-52 (2001).

4. Sangouard, N. \& Zbinden, H. What are single photons good for? J. Mod. Opt. 59, 1458-1464 (2012).

5. Gatto Monticone, D. et al. Beating the Abbe diffraction limit in confocal microscopy via nonclassical photon statistics. Phys. Rev. Lett. 113, 143602 (2014).

6. Lombardi, P. et al. A molecule-based single-photon source applied in quantum radiometry. Adv. Quantum Technol. 3, 1900083 (2019).

7. Duan, L.-M. \& Kimble, H. J. Scalable photonic quantum computation through cavity-assisted interactions. Phys. Rev. Lett. 92, 127902 (2004)

8. Basché, T., Moerner, W. E., Orrit, M. \& Wild, U. P. Single-Molecule Optical Detection, Imaging and Spectroscopy (VCH, 1997).

9. Plakhotnik, T., Donley, E. A. \& Wild, U. P. Single-molecule spectroscopy. Annu. Rev. Phys. Chem. 48, 181-212 (1997)

10. Kozankiewicz, B. \& Orrit, M. Single-molecule photophysics, from cryogenic to ambient conditions. Chem. Soc. Rev. 43, 1029-1043 (2014).

11. Orrit, M. \& Bernard, J. Single pentacene molecules detected by fluorescence excitation in a p-terphenyl crystal. Phys. Rev. Lett. 65, 2716-2719 (1990)

12. Weiss, S. Fluorescence spectroscopy of single biomolecules. Science 283, 1676-1683 (1999).

13. Zürner, A., Kirstein, J., Döblinger, M., Bräuchle, C. \& Bein, T. Visualizing single-molecule diffusion in mesoporous materials. Nature 450, 705-708 (2007).

14. Buchler, B. C., Kalkbrenner, T., Hettich, C. \& Sandoghdar, V. Measuring the quantum efficiency of the optical emission of single radiating dipoles using a scanning mirror. Phys. Rev. Lett. 95, 063003 (2005).

15. Wang, D. et al. Turning a molecule into a coherent two-level quantum system. Nat. Phys. 15, 483-489 (2019).

16. Basché, T., Moerner, W. E., Orrit, M. \& Talon, H. Photon antibunching in the fluorescence of single dye molecule trapped in a solid. Phys. Rev. Lett. 69, 1516-1519 (1992).
17. Wrigge, G., Gerhardt, I., Hwang, J., Zumofen, G. \& Sandoghdar, V. Efficient coupling of photons to a single molecule and the observation of its resonance fluorescence. Nat. Phys. 4, 60-66 (2008).

18. Gerhardt, I. et al. Coherent state preparation and observation of Rabi oscillations in a single molecule. Phys. Rev. A 79, 011402(R) (2009).

19. Lettow, R. et al. Quantum interference of tunably indistinguishable photons from remote organic molecules. Phys. Rev. Lett. 104, 123605 (2010).

20. Trebbia, B., Tamarat, P. \& Lounis, B. Indistinguishable near-infrared single photons from an individual organic molecule. Phys. Rev. A 82, 063803 (2010)

21. Nonn, T \& Plakhotnik, T. Fluorescence excitation spectroscopy of vibronic transitions in single molecules. Chem. Phys. Lett. 336, 97-104 (2001).

22. Kiefer, W., Rezai, M., Wrachtrup, J. \& Gerhardt, I. An atomic spectrum recorded with a single molecule light source. Appl. Phys. B 122, 38 (2016).

23. De Martini, F., Di Giuseppe, G. \& Marrocco, M. Single-mode generation of quantum photon states by excited single molecules in a microcavity trap. Phys. Rev. Lett. 76, 900-903 (1996).

24. Ambrose, W. P. et al. Fluorescence photon antibunching from single molecules on a surface. Chem. Phys. Lett. 269, 365-370 (1997).

25. Lounis, B. \& Moerner, W. Single photons on demand from a single molecule at room temperature. Nature 407, 491-493 (2000).

26. Senellart, P., Solomon, G. \& White, A. High-performance semiconductor quantum-dot single-photon sources. Nat. Nanotechnol. 12, 1026-1039 (2017).

27. Lenzini, F., Gruhler, N., Walter, N. \& Pernice, W. H. P. Diamond as a platform for integrated quantum photonics. Adv. Quantum Technol. 1, 1800061 (2018).

28. Chakraborty, C., Vamivakas, N. \& Englund, D. Advances in quantum light emission from 2D materials. Nanophotonics 8, 2017-2032 (2019).

29. Rezai, M., Wrachtrup, J. \& Gerhardt, I. Coherence properties of molecular single photons for quantum networks. Phys. Rev. X 8, 031026 (2018).

30. Nicolet, A. A. L., Hofmann, C., Kol'chenko, M., Kozankiewicz, B. \& Orrit, M. Single dibenzoterrylene molecules in an anthracene crystal: spectroscopy and photophysics. ChemPhysChem 8, 1215-1220 (2007).

31. Toninelli, C. et al. Near-infrared single-photons from aligned molecules in ultrathin crystalline films at room temperature. Opt. Express 18, 6577-6582 (2010).

32. Polisseni, C. et al. Stable, single-photon emitter in a thin organic crystal for application to quantum-photonic devices. Opt. Express 24, 5615-5627 (2016).

33. Trebbia, J.-B., Ruf, H., Tamarat, P. \& Lounis, B. Efficient generation of near-infrared single photons from the zero-phonon line of a single molecule. Opt. Express 17, 23986 (2009).

34. Chu, X., Götzinger, S. \& Sandoghdar, V. A single molecule as a high-fidelity photon gun for producing intensity-squeezed light. Nat. Photon. 11, 58-62 (2017)

35. Colautti, M. et al. A $3 \mathrm{D}$ polymeric platform for photonic quantum technologies. Adv. Quantum Technol. 3, 2000004 (2020).

36. Lee, K. et al. A planar dielectric antenna for directional single-photon emission and near-unity collection efficiency. Nat. Photon. 5, 166-169 (2011)

37. Zirkelbach, J. et al. Partial cloaking of a gold particle by a single molecule. Phys. Rev. Lett. 125, 123603 (2020).

38. Siyushev, P., Stein, G., Wrachtrup, J. \& Gerhardt, I. Molecular photons interfaced with alkali atoms. Nature 509, 66-70 (2014).

39. Kiraz, A. et al. Indistinguishable photons from a single molecule. Phys. Rev. Lett. 94, 223602 (2005).

40. Lombardi, P. et al. Indistinguishable photons on demand from an organic dye molecule. Preprint at https://arxiv.org/abs/2102.13055 (2021).

41. Wild, U. P., Guettler, F., Pirotta, M. \& Renn, A. Single molecule spectroscopy: Stark effect of pentacene in p-terphenyl. Chem. Phys. Lett. 193, 451-455 (1992).

42. Rezai, M., Wrachtrup, J. \& Gerhardt, I. Polarization-entangled photon pairs from a single molecule. Optica 6, 34-40 (2019).

43. Brunel, C., Tamarat, P., Lounis, B., Woehl, J. C. \& Orrit, M. Stark effect on single molecules of dibenzanthanthrene in naphthalene crystal and in a n-hexadecane Shpol'skii matrix. J. Phys. Chem. A 103, 2429-2434 (1999).

44. Moradi, A., Ristanović, Z., Orrit, M., Deperasińska, I. \& Kozankiewicz, B. Matrix-induced linear Stark effect of single dibenzoterrylene molecules in 2,3-dibromonaphthalene crystal. Chem. Phys. Chem. 20, 55-61 (2019).

45. Schaedler, K. et al. Electrical control of lifetime-limited quantum emitters using 2D materials. Nano Lett. 19, 3789-3795 (2019).

46. Colautti, M. et al. Laser-induced frequency tuning of Fourier-limited single-molecule emitters. ACS Nano 14, 13584-13592 (2020).

47. Lombardi, P. et al. Photostable molecules on chip: integrated sources of nonclassical light. ACS Photon. 5, 126-132 (2018).

48. Hwang, J. et al. A single-molecule optical transistor. Nature 460, 76-80 (2009).

49. Mollow, B. R. Stimulated emission and absorption near resonance for driven systems. Phys. Rev. A 5, 2217-2222 (1972). 
50. Lounis, B., Jelezko, F. \& Orrit, M. Single molecules driven by strong resonant fields: hyper-Raman and subharmonic resonances. Phys. Rev. Lett. 78, 3673-3676 (1997).

51. Lezama, A., Zhu, Y., Kanskar, M. \& Mossberg, T. W. Radiative emission of driven two-level atoms into the modes of an enclosing optical cavity: the transition from fluorescence to lasing. Phys. Rev. A 41, 1576-1581 (1990).

52. Maser, A., Gmeiner, B., Utikal, T., Götzinger, S. \& Sandoghdar, V. Few-photon coherent nonlinear optics with a single molecule. Nat. Photon. 10, 450-453 (2016).

53. Leuchs, G. \& Sondermann, M. Light-matter interaction in free space. J. Mod. Opt. 60, 36-42 (2013).

54. Barnes, W. L. et al. Solid-state single photon sources: light collection strategies. Eur. Phys. J. D 18, 197-210 (2002).

55. Checcucci, S. et al. Beaming light from a quantum emitter with a planar optical antenna. Light. Sci. Appl. 6, e16245 (2017).

56. Skoff, S. M., Papencordt, D., Schauffert, H., Bayer, B. C. \& Rauschenbeutel, A. Optical-nanofiber-based interface for single molecules. Phys. Rev. A 97, 043839 (2018).

57. Stein, G., Bushmakin, V., Wang, Y., Schell, A. W. \& Gerhardt, I. Narrow-band fiber-coupled single-photon source. Phys. Rev. Appl. 13, 054042 (2020).

58. Faez, S., Türschmann, P., Haakh, H. R., Götzinger, S. \& Sandoghdar, V. Coherent interaction of light and single molecules in a dielectric nanoguide Phys. Rev. Lett. 113, 213601 (2014).

59. Ferrari, S., Schck, C. \& Pernice, W. Waveguide-integrated superconducting nanowire single-photon detectors. Nanophotonics 7, 1725-1728 (2018).

60. Türschmann, P. et al. Chip-based all-optical control of single molecules coherently coupled to a nanoguide. Nano Lett. 17, 4941-4945 (2017).

61. Boissier, S. et al. Coherent characterisation of a single molecule in a photonic black box. Nat. Commun. 12, 706 (2021).

62. Hwang, J. \& Hinds, E. A. Dye molecules as single-photon sources and large optical nonlinearities on a chip. New J. Phys. 13, 085009 (2011).

63. Rivoire, K. et al. Lithographic positioning of fluorescent molecules on high-Q photonic crystal cavities. Appl. Phys. Lett. 95, 123113 (2009).

64. Ciancico, C. et al. Narrow line width quantum emitters in an electron-beam-shaped polymer. ACS Photon. 6, 3120-3125 (2019).

65. Shi, Q. et al. Wiring up pre-characterized single-photon emitters by laser lithography. Sci. Rep. 6, 31135 (2016).

66. Hail, C. U. et al. Nanoprinting organic molecules at the quantum level. Nat. Commun. 10, 1880 (2019).

67. Kewes, G. et al. A realistic fabrication and design concept for quantum gates based on single emitters integrated in plasmonic-dielectric waveguide structures. Sci. Rep. 6, 28877 (2016).

68. Grandi, S. et al. Hybrid plasmonic waveguide coupling of photons from a single molecule. APL Photon. 4, 086101 (2019).

69. Rattenbacher, D. et al. Coherent coupling of single molecules to on-chip ring resonators. New J. Phys. 21, 062002 (2019).

70. Toninelli, C. et al. A scanning microcavity for in situ control of single-molecule emission. Appl. Phys. Lett. 97, 021107 (2010).

71. Wang, D. et al. Coherent coupling of a single molecule to a scanning Fabry-Perot microscavity. Phys. Rev. X 7, 021014 (2017).

72. Lodahl, P., Mahmoodian, S. \& Stobbe, S. Interfacing single photons and single quantum dots with photonic nanostructures. Rev. Mod. Phys. 87, 347-400 (2015).

73. Zhang, J. L. et al. Strongly cavity-enhanced spontaneous emission from silicon-vacancy centers in diamond. Nano Lett. 18, 1360-1365 (2018).

74. Schwartz, T., Hutchison, J. A., Genet, C. \& Ebbesen, T. W. Reversible switching of ultrastrong light-molecule coupling. Phys. Rev. Lett. 106, 196405 (2011)

75. Haakh, H. R., Faez, S. \& Sadoghdar, V. Polaritonic normal-mode splitting and light localization in a one-dimensional nanoguide. Phys. Rev. A 94, 053840 (2016).

76. Kim, J., Yang, D., Oh, S.-H. \& An, K. Coherent single-atom superradiance. Science 359, 662-666 (2018).

77. Hettich, C. et al. Nanometer resolution and coherent optical dipole coupling of two individual molecules. Science 298, 385-389 (2002).

78. Gerhardt, I., Wrigge, G., Hwang, J., Zumofen, G. \& Sandoghdar, V. Coherent nonlinear single molecule microscopy. Phys. Rev. A 82, 063823 (2010).

79. Orrit, M., Bernard, J., Zumbusch, A. \& Personov, R. Stark effect on single molecules in a polymer matrix. Chem. Phys. Lett. 196, 595-600 (1992).

80. Brunel, C., Lounis, B., Tamarat, P. \& Orrit, M. Rabi resonances of a single molecule driven by rf and laser fields. Phys. Rev. Lett. 81, 2679-2682 (1998).

81. Caruge, J. M. \& Orrit, M. Probing local currents in semiconductors with single molecules. Phys. Rev. B 64, 205202 (2001).

82. Kador, L., Latychevskaia, T., Renn, A. \& Wild, U. P. Radio-frequency Stark effect modulation of single-molecule lines. J. Lumin. 86, 189-194 (2000).

83. Plakhotnik, T. Sensing single electrons with single molecules. J. Lumin. 127, 235-238 (2007).
84. Faez, S., van der Molen, S. \& Orrit, M. Optical tracing of multiple charges in single-electron devices. Phys. Rev. B 90, 205405 (2014).

85. Plakhotnik, T. Single-molecule dynamic triangulation. ChemPhysChem 7, 1699-1704 (2006)

86. Croci, M., Müschenborn, H.-J., Güttler, F., Renn, A. \& Wild, U. P. Single molecule spectroscopy: pressure effect on pentacene in $p$-terphenyl. Chem. Phys. Lett. 212, 71-77 (1993).

87. Kol'chenko, M. A. et al. Single molecules detect ultra-slow oscillators in a molecular crystal excited by ac voltages. New J. Phys. 11, 023037 (2009).

88. Tian, Y., Navarro, P. \& Orrit, M. Single molecule as a local acoustic detector for mechanical oscillators. Phys. Rev. Lett. 113, 135505 (2014).

89. Puller, V., Lounis, B. \& Pistolesi, F. Single molecule detection of nanomechanical motion. Phys. Rev. Lett. 110, 125501 (2013).

90. Dutreix, C., Avriller, R., Lounis, B. \& Pistolesi, F. Two-level system as topological actuator for nano-mechanical modes. Phys. Rev. Res. 2, 023268 (2020).

91. Bauer, M. \& Kador, L. Zeeman effect of single-molecule lines. Chem. Phys. Lett. 407, 450-453 (2005).

92. Wrachtrup, J., von Borczyskowski, C., Bernard, J., Orrit, M. \& Brown, R. Optical detection of magnetic resonance in a single molecule. Nature 363, 244-245 (1993).

93. Köhler, J. et al. Magnetic resonance of a single molecular spin. Nature 363, 242-244 (1993).

94. Brouwer, A. C. J., Groenen, E. J. J. \& Schmidt, J. Detecting magnetic resonance through quantum jumps of single molecules. Phys. Rev. Lett. 80, 3944 (1998).

95. Gaudreau, L. et al. Universal distance-scaling of nonradiative energy transfer to graphene. Nano Lett. 13, 2030-2035 (2013).

96. Mazzamuto et al. Single-molecule study for a graphene-based nano-position sensor. New J. Phys. 16, 113007 (2014).

97. Das, S., Elfving, V. E., Faez, S. \& Sorensen, A. S. Interfacing superconducting qubits and single optical photons using molecules in waveguides. Phys. Rev. Lett. 118, 140501 (2017).

98. Muschik, C. A. et al. Harnessing vacuum forces for quantum sensing of graphene motion. Phys. Rev. Lett. 112, 223601 (2014).

99. Carusotto, J. \& Ciuti, C. Quantum fluids of light. Rev. Mod. Phys. 85, 299-366 (2013).

100. Sandoghdar, V. Nano-optics in $2020 \pm 20$. Nano Lett. 20, 4721-4723 (2020).

101. Philippe Roelli, P., Galland, C., Piro, N. \& Kippenberg, T. J. Molecular cavity optomechanics as a theory of plasmon-enhanced Raman scattering. Nat. Nanotechnol. 11, 164-169 (2016).

102. Clear, C. et al. Phonon-induced optical dephasing in single organic molecules. Phys. Rev. Lett. 124, 153602 (2020).

103. Reitz, M. et al. Molecule-photon interactions in phononic environments. Phys. Rev. Res. 2, 033270 (2020).

104. Wasielewski, M. R. et al. Exploiting chemistry and molecular systems for quantum information science. Nat. Rev. Chem. 4, 490-504 (2020).

105. Bonizzoni, C. et al. Storage and retrieval of microwave pulses with molecular spin ensembles. npj Quantum Inf. 6, 68 (2020).

106. Bayliss, S. L. et al. Optically addressable molecular spins for quantum information processing. Science 370, 1309-1312 (2020).

107. Pazzagli, S. et al. Self-assembled nanocrystals of polycyclic aromatic hydrocarbons show photostable single-photon emission. ACS Nano 12, 4295-4303 (2018).

108. Hildner, R., Brinks, D., Nieder, J. B., Cogdell, R. J. \& van Hulst, N. F. Quantum coherent energy transfer over varying pathways in single light-harvesting complexes. Science 340, 1448-1451 (2013).

109. Liebel, M., Toninelli, C. \& van Hulst, N. F. Room-temperature ultrafast nonlinear spectroscopy of a single molecule. Nat. Photon. 12, 45-49 (2018).

110. Zhang, L. et al. Electrically driven single-photon emission from an isolated single molecule. Nat. Commun. 8, 580 (2017).

111. Nicolet, A., Kol'chenko, M., Kozankiewicz, B. \& Orrit, M. Intermolecular intersystem-crossing in single-molecule spectroscopy, terrylene in anthracene crystals. J. Chem. Phys. 124, 164711 (2006).

112. Ambrose, W. P., Basché, T. \& Moerner, W. E. Detection and spectroscopy of single pentacene molecules in a $p$-terphenyl crystal by means of fluorescence excitation. J. Chem. Phys. 95, 7150-7163 (1991).

113. Sola, M. Forty years of Clar's aromatic $\pi$-sextet rule. Front. Chem. https:// doi.org/10.3389/fchem.2013.00022 (2013).

114. Avlasevich, Y. \& Müllen, K. Dibenzopentarylenebis(dicarboximide)s: novel near-infrared absorbing dyes. Chem. Commun. 42, 4440-4442 (2006).

115. Langhals, H., Zgela, D. \& Lüling, R. Sexterrylenetetracarboxylic bisimides: NIR dyes. J. Org. Chem. 80, 12146-12150 (2015).

116. Henry, B. R. \& Siebrand, W. in Organic Molecular Photophysics (ed. Birks, J. B.) Ch. 4 (Wiley, 1973)

117. Sakamoto, Y. et al. Perfluoropentacene and perfluorotetracene: syntheses, crystal structures, and FET characteristics. Mol. Cryst. Liq. Cryst. 444, 225-232 (2006). 
118. Yamada, $\mathrm{H}$. Photochemical synthesis of pentacene and its derivatives. Chem. Eur. J. 11, 6212-6220 (2005).

119. Watanabe, M. et al. The synthesis, crystal structure and charge transport properties of hexacene. Nat. Chem. 4, 574-578 (2012).

120. Jancarik, A., levet, G. \& Gourdon, A. A practical general method for the preparation of long acenes. Chem. Eur. J. 25, 2366-2374 (2019).

121. Lounis, B. \& Orrit, M. Single-photon sources. Rep. Prog. Phys. 68 , 1129-1179 (2005).

122. Schofield, R. C. et al. Efficient excitation of dye molecules for single photon generation. J. Phys. Commun. 2, 115027 (2018).

123. Steinberg, A. M., Kwiat, P. G. \& Chiao, R. Y. Dispersion cancellation in a measurement of the single-photon propagation velocity in glass. Phys. Rev. Lett. 68, 2421-2424 (1992).

124. Bennet, C. H. et al. Teleporting an unknown quantum state via dual classical and Einstein-Podolsky-Rosen channels. Phys. Rev. Lett. 70, 1895-1899 (1993).

125. Bouwmeester, D. et al. Experimental quantum teleportation. Nature 390, 575-579 (1997).

126. Grandi, S. et al. Quantum dynamics of a driven two-level molecule with variable dephasing. Phys. Rev. A 94, 063839 (2016).

\section{Acknowledgements}

This project has received funding from the EraNET Cofund Initiatives QuantERA within the European Union's Horizon 2020 research and innovation programme grant agreement no. 731473 (project ORQUID). A.S.C. acknowledges a University Research
Fellowship from the Royal Society (UF160475) and funding from the EPSRC (EP/ P030130/1, EP/P01058X/1 and EP/R044031/1). W.H.P. and I.G. acknowledge funding from the Deutsche Forschungs gemeinschaft (DFG) - Projektnummer 332724366 and GE2737/5-1, respectively. F.H.L.K. and A.R.-P. acknowledge support from the Government of Spain (FIS2016-81044; Severo Ochoa CEX2019-000910-S), Fundació Cellex, Fundació Mir-Puig, and Generalitat de Catalunya (CERCA, AGAUR, SGR 1656). Furthermore, the research leading to these results has received funding from the European Union's Horizon 2020 under grant agreement no. 820378 (Quantum Flagship). We thank A. Moradi for discussions and NWO (The Dutch Research Council) for funding of his PhD grant on sensing of single charges. C.T. thanks A. Renn for always useful discussions.

\section{Competing interests}

The authors declare no competing interests.

\section{Additional information}

Correspondence should be addressed to C.T.

Peer review information Nature Materials thanks the anonymous reviewers for their contribution to the peer review of this work.

Reprints and permissions information is available at www.nature.com/reprints.

Publisher's note Springer Nature remains neutral with regard to jurisdictional claims in published maps and institutional affiliations.

(c) Springer Nature Limited 2021 